\title{
ABSTRACTS COLLECTION ACNP 57th Annual Meeting: Keyword Index
}

(2R,6R)-HNK . . . . . . . . . . . . . . . . . . . . . . . . . . . . T148

(S)-Norketamine $\ldots \ldots \ldots \ldots \ldots \ldots \ldots \ldots \ldots$ 42.1, W131

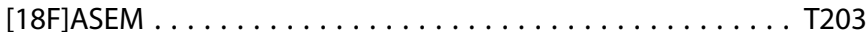

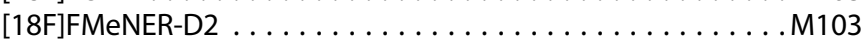

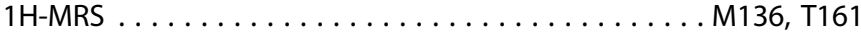

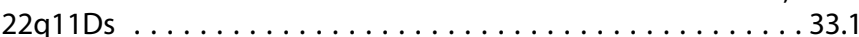

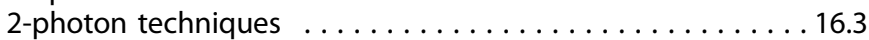

5 -HT1A receptors . ...................W137

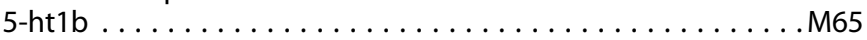

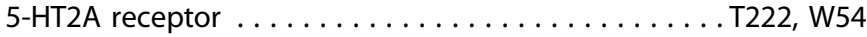

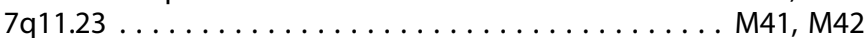

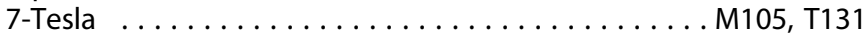

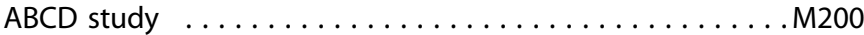

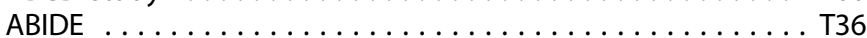

Abstinence ........... M248, T247, T257, W252, W257

Abuse Liability . . . . . . . . . . . . . . M265, M269

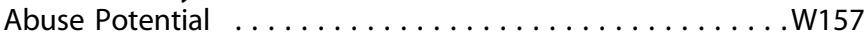

Acamprosate . . . . . . . . . . . . . . . . . . . . . . M234

Accumbens . . . . . . . . . . . . . . . . . . . . . . . . . . . . . W284

Acetate . . . . . . . . . . . . . . . . . . W263

Acetylcholine .....53, 53.1, 53.3, 53.4, M68, M129, W55, W282

Acetylcholine esterase inhibitors, muscarinic receptors,

dementia . . . . . . . . . . . . . . . . . . . . . . . . . M236

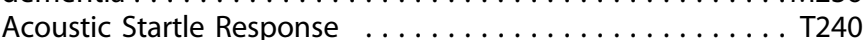

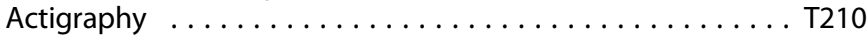

Actin Remodeling . . . . . . . . . . . . . . . . . . . . . . T245

Activity-Regulated Cytoskeletal Associated Protein (Arc) ... T230

Acute and Chronic Stress ....... . 1.2, 1.3, 20.2, 42.2, 51.1, W30

Acute Stress ....... M86, M125, M165, M167, T32, T232, T239

Adaptive Behavior . . . ...............W162

Addiction . ........9.9.3, 30, 40.3, 41.1, 41.2, 50.3, 50.4, 53.2

M281, T52, T255, T271, T273, T275, W44, W238

Addiction Circuitry . . . . . . 23.3, 41.3, 45.3, M231, T233, T270

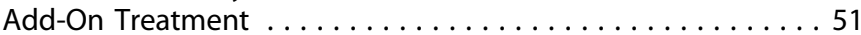

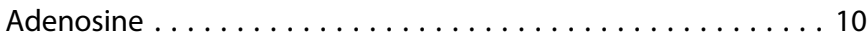

Adenosine A2A Receptor ....................W239

Adenosine deaminases acting on RNA .............M178

Adenosine Triphosphate (ATP) . . . . . . . . . . . . W83

Adjunctive Brexpiprazole . . . . . . . . . . . . . . . . . . . . . T141

Adolescence .... 39.3, 39.4, 48, 54, M28, M29, M45, M56, M88, M230, M257, M267, M275, T87, T88, W50, W268

Adolescent ......2.3, M33, M47, M98, M225, W46, W114, W199

Adolescent Alcohol . . . . . . . . . . . . M227, T234, T264, W268

Adolescent Alcohol Exposure . . . . . . . . . . . . . . . . . . M7

Adolescent Alcohol Use . . . . . . . . . . . . . . . . M236, W39

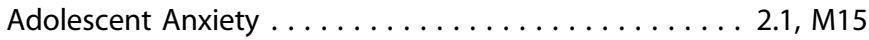

Adolescent Binge Drinking . . . . . . . . . . . . . . . . . . . . . . M247

Adolescent Brain and Cognitive Development Study . . .24, 24.1,

$24.3, \mathrm{M} 34$

Adolescent Depression ........... M32, M48, T26, T136, W46,

W117, W124, W125

Adolescent Development . . . . . . . . . . . . . . . . . . W281

Adolescent Stress . . . . . . . . . . . . . . . . . T60, W121

Adolescents . . . . . . . . . . . . . . . . . . . . . M228, T84

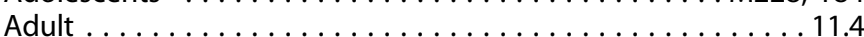

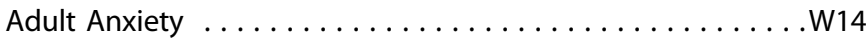

Adult Hippocampal Neurogenesis . . . . . . . . . . . . . . W153

Adult Stem Cells . . . . . . . . . . . . . . . . . . T254, W212

Adult-Child Interactions . . . . . . . . . . . . . . T93

Advantages of the Combination of Therapies . . . . . . . M183

Adverse Childhood Experiences (ACE) . . . . . . . . M25, T159

Aerobic Exercise ......................W211

Affective Components of Pain . . . . . . . . . . . . . 16.4 Affective Disorders . . . . . . . . . . . . . M97, W71, W258

Affective Instability . . . . . . . . . . . . . . 46.1

Affective Neuroscience . . . . . . . . . . . T18, T91, W99

Age Effects . . . . . . . . . . . . . . . . . . . . . . . . . W277 Aggression ...........M16, M282, T83, T216, W28, W74

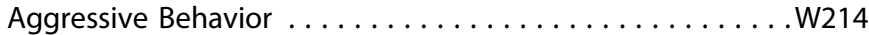

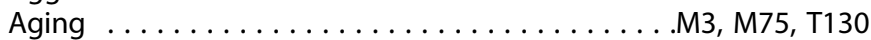

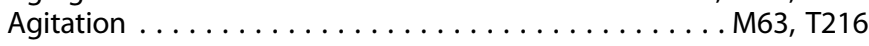

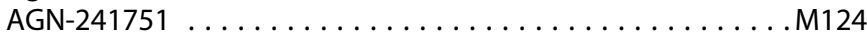

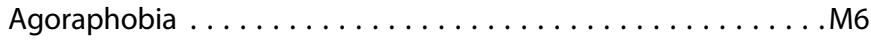

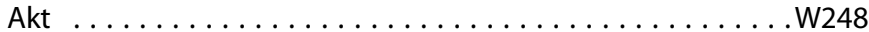

Alcohol ... . . 35.2, T48, T55, T57, T176, T237, T246, T260, T285,

W254, W276

Alcohol Abuse ..............................W263

Alcohol and Substance Use Disorders .......M35, M257, M259,

M280, T227, T258, T280, W262

Alcohol Consumption ...................T53, T225, T272

Alcohol Dependence .... . T53, T235, T272, W277, W279, W280

Alcohol Exposure . . . . . . . . . . . . . . . . . . . . . M263

Alcohol Intake . . . . . . . . . . . . . . . . . W231

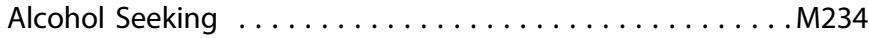

Alcohol Sensitivity . . . . . . . . . . . . . . . . . . . . . . . . M251

Alcohol Use Disorder . . . . . . . M7, M261, T53, T56, T78, T240,

T247, T253, W249, W258, W271

Alcohol Withdrawal ...................W263

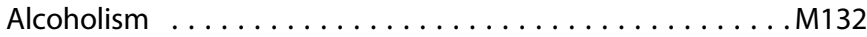

Alcohol-Seeking Behavior . . . . . . . . . . . . . . . . . . W239

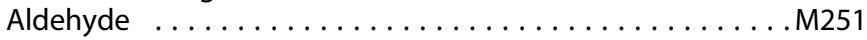

Aldosterone .......................W271

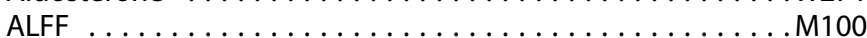

Allopregnanolone ................ 8.3, M242, T14

Alpha2 Adrenergic Receptors . . . . . . . . . . . . . . . M222, T216

Alpha7 Nicotinic Acetylcholine Receptor . . . . . . . . . . T203

Alpha-7 Nicotinic Acetylcholine Receptor M134, T41, T188, W162

Altitude . . . . . . . . . . . . . . . . . . . . . . . . . W259

Alzheimer's Disease . . . . . . . 43.3, 53, 53.4, M4, M40, M53,

M220, T253, W52

AMPA glutamate receptors ...........27.3, M119, T271

AMPA Receptor . ............ 20.1, M148, T271, W208 Amphetamine ............. M240, W47, W189, W219, W281 Amygdala . . . . . 17, 17.3, 45.4, 49.4, 53.1, 54, 54.3, M19, M166, M252, T8, T10, T12, T104, T135, T159, T173, T175, T234, T279, T284, W155, W283

Amygdala Ablation . . . . . . . . . . . . . . . W167 Amygdala-Based Networks . . . . . . . . . . . . . . 35.1, W154

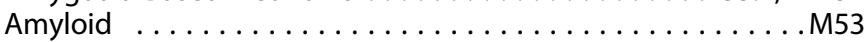
Analgesia . . . . . . . . . . . . . . . . . . . M270, T157

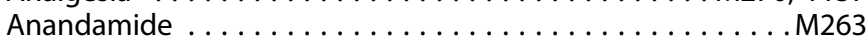

Anger ............................... M169 
Anhedonia . . ...12.3, 35, 35.1, 35.2, 35.3, 35.4, 41.2, M66, M73, M91, T103, T152, W97, W252

Animal Model . . . . . . . . . . . . . . . . . . . . . M270, M273, W194

Animal Research . . . . . . . . . . . . . . . . . . . . W266

ANK3 . . . . . . . . . . . . . . . . . . . . . . T102, T109

Anorexia Nervosa . . . . . . . . . . 15, 15.1, 15.2, M59, T98, T99,

T101, W60, W63

Anterior Cingulate Cortex (ACC) . . . . M80, M87, T56, W62, W269

Anterior Prefrontal Cortex . . . . . . . . . . . . . . . . . T195

Antibody . . . . . . . . . . . . . . . . . . . . M52, M186

Antidepressant ......34.2, 42, 42.1, T31, T69, T125, W89, W96,

W137, W153

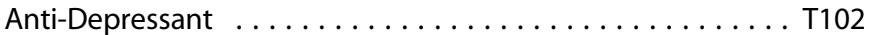

Antidepressant Effect . . . . . . . . . . . . . . . . M148

Antidepressant Response . . . . . . . . M2, T29, W132, W138

Antidepressants ..............20, M72, M76, T123

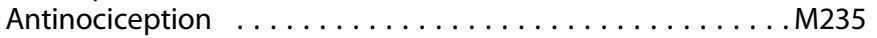

Antipsychotic .................M184, T44, T184, W199

Antipsychotic Medication . . . . . . . . . . . . . . M196

Antipsychotic Response . . . . . . . . . . . . . . . . T186

Antipsychotic Treatment .............M210, W201, W207

Antipsychotic Unduced Weight Gain . . . . . . . . . . W151

Antipsychotic-Associated Obesity . . . . . . . . . . . . M38

Antipsychotic-Naïve First-Episode Schizophrenia . . . . . . 25, 25.2,

M203, W203

Antipsychotic-Naive Schizophrenia . . . . . . . . . . 25, 25.4, 29.2

Antipsychotics . ..........25.4, 38, M217, T43, T46, T213, W184, W193, W220, W224

Anti-Psychotics ..........................W225

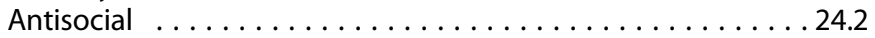

Anxiety . . . . . . . . 2, 48, 48.1, M6, M13, M22, M23, M73, M284, T8, T10, T72, T73, T78, T80, T81, T105, T155, T223, T234, W94

Anxiety Circuitry . . . . . . . . . . . . . . . . . 45.3, M10, M12

Anxiety Disorders . . . . . . . . . . .53.2, T5, T12, W15

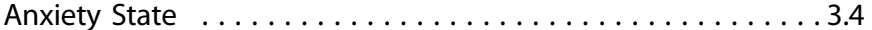

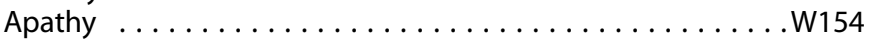

Appetite ......................... 15.3

Approach/Avoidance .................... T81

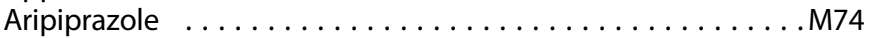

Aripiprazole Lauroxil . . . . . . . . . . . . . . . . . . . .W186

Aripiprazole Once-Monthly $400 \mathrm{Mg}$ (AOM 400) . . . . . . . W184

Arousal . . . .........................W27

Asperger's Spectrum Disorder . . . . . . . . . . . . M170

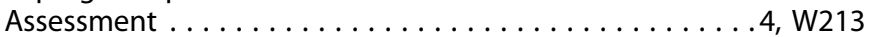

Associative Learning . . . . . . . . . . . . . . 16.1, W79, W255

Astrocyte ..............26, T106, T281, W83, W152

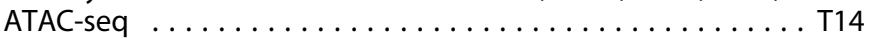

A-to-I RNA-editing . . . . . . . . . . . . . . . . . . . . . . M178

At-Risk . . . . . . . . . . . . . . . . . . . . . . T132

Attachment . . . . . . . . . . . . . . . . . . . . 37.3

Attention . . . . . . . . . 53, 53.3, M31, M247, T58, T87,

T92, W162, W189

Attention-Deficit/Hyperactivity Disorder (ADHD) . .M36, T16, T18, T58, T86, T90, W29, W47, W61, W65, W156, W157

Atypical Antipsychotics . . . . . . . . . . . . .T145, T180 Auditory Deficits In Schizophrenia .........M175, M181, T205 Auditory Hallucinations . . . . . . . . . . . . . . T76, W185 Auditory Short-Term Memory . . . . . . . . . . . . . . . . M173 Autism Spectrum Disorder (ASD) . . . . . . . 11.1, 13, 13.1, 21, 26.1, 26.3, 27.1, 39.2, 43.4, 51.2, M144, M170, M216, T15, T35, T36, T82, T94, T96, T161, W33, W36, W45, W143, W215

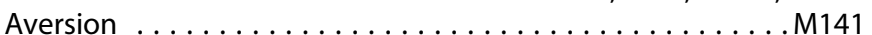

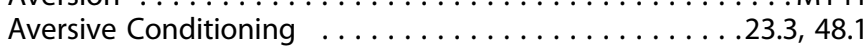

Avoidance ....................... M25, T75, W283

Axons ... . . . . . . . . . . . . . . . . . . . . . . . . 54.1
Baby Schema . . . . . . . . . . . . . . . . . . T252

Baclofen .........................W276

Balb/c mouse . . . . . . . . . . . . . . . . . . . T96

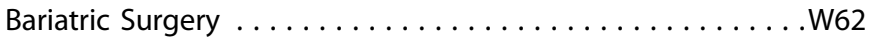

Basal Ganglia .......... . . . . . . . . . M58, T101, W79

Basolateral Amygdala . . . . . . . . . . . . . . . 15.1, M19

Bayesian Modeling ...................... M18 BDNF . . . . . . T65, T116, T146, T234, W6, W10, W175, W211

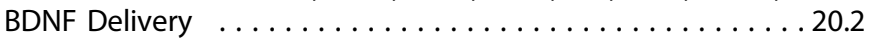

BDNF Val66Met . . . . . . . . . . . . . . . . T116, W78

Bed Nucleus of the Stria Terminalis . . . . . . . . . . . . . M284

Behavior . . . . . . . . . . . T70, T169, T178, W226

Behavioral . . . . . . . . . . . . . . . . . . . W108, W119

Behavioral Analysis ....................W230

Behavioral Despair ...................... T103

Behavioral Pharmacology . . . . . . . . . . . . . . . . T59, W25

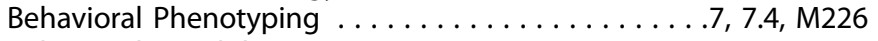

Behavioral Variability ....................6

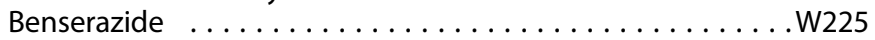

Benzodiazepines ........................W127

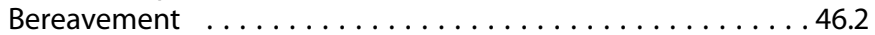

Beta Arrestin . . . . . . . . . . . . . . .

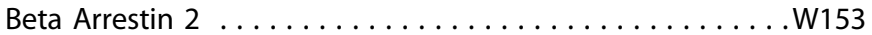

Betahisitine .........................W220

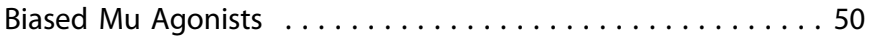

Bile Acids ........................ 38

Binge Drinking .............. M247, M277, T244, T258

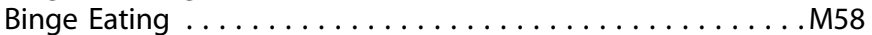

Binge Eating Disorder . . . . . . . . . . 15.2, M55, T97, W61, W64

Bioinformatics ................. 11.4, W37, W59

Biomarker . . . . . . . 12.1, 20.3, M107, M130, M150, T4, T29,

T114, T136, T210, T254, W1, W16, W20, W142

Biomarkers for Risk Assessment . . . . . ....M114, W21, W52

Biotypes . . . . . . . . . . . . . . . . . . 25.3, 56.3

Bipolar Depression . . . . . . . . . . . . T95, T119, W130

Bipolar Disorder . ...........11.1, 25, 25.3, 44, 44.1, 44.4,

54.4, M68, M70, M81, M82, M85,

M90, M93, M95, M104, M116, M201, T24, T109,

T114, T122, T124, T126, T132, T204, W82,

W90, W93, W108, W111, W114, W119, W129

Bipolar I Depression . . . . . . . . . . . . . . ..W134 Bipolar I Disorder ................44.1, W130, W182

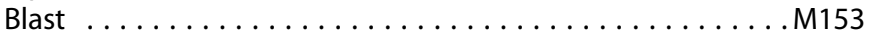

Blood-Brain-Barrier . . . . . . . . . . . . . . . . . . . . . M52

Body Mass Index .................... T98

BOLD Imaging $\ldots \ldots \ldots \ldots \ldots \ldots \ldots \ldots \ldots . \ldots \ldots . \ldots \ldots$

Borderline Personality Disorder ......4.3, 17.3, 46, 46.1, M169,

M170, M171

Brain . . . . . . . . . . . . . . . . . . M61, T221, W203, W162

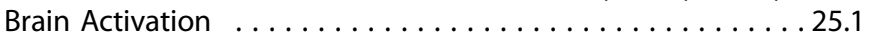

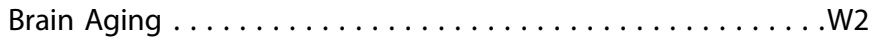

Brain Based Markers for Depression . . . . . . . . . . . . . . . . M91

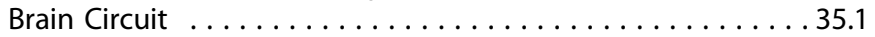

Brain Connectivity . . . . . . . . . . . . . . . 29.2, M40

Brain Connectome . . . . . . . . . . . . . . . . . . . . . . . 56.2

Brain Development . . . . . . 24, 39.1, 48.1, M49, T20, W18, W41

Brain Glucose Metabolism . . . . . . . . . . . . . . . . . . . M261

Brain Imaging . .............. 18.1, 46.1, T190, W63

Brain Insulin Resistance . . . . . . . . . . . . . . . . . . M197

Brain MRI . . . . . . . . . . . . . . . . . . . . . . M109

Brain Networks . . . . . . . . . . . . . . . . . . 25.1

Brain Organoids . . . . . . . . . . . . . . . . . . M241, T15

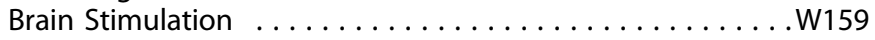

Brain Stress . . . . . . . . . . . . . . . . . . . . . T259

Brain Structural Connectivity ................... M135

Brain Structure ....................24.1, M15

Brain Transcription . . . . . . . . . . . . . . . . . . . . . . 44.4 
Brain Volumes . . . . . . . . . . . . . . . . . . . . . . . . . M250

Brainstem ........................ T48

Brexanolone ....................... 8.3

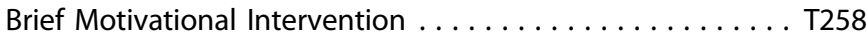

Bulimia Nervosa . . . . . . . . . . . . . . . . . . . . . . M58

Buprenorphine ............... T259, W85, W136

Buprenorphine/samidorphan . . . . . . . . . M118, T138

Buprenorphine-Naloxone . . . . . . . . . T187, T243, W250

burden of Illness ..................... T113

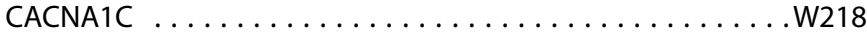

Calcium . . . . . . . . . . . . . . . . . . . . . . . . . . . . M146

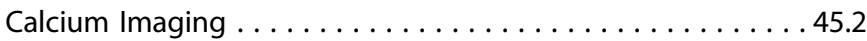

Callous-Unemotional . . . . . . . . . . . . . . . . 24.2

CaMKII . . . . . . . . . . . . . . . . . . . . . . T178, W248

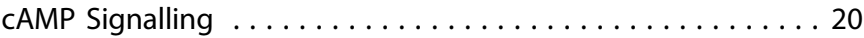

Cannabidiol ................... 21, 32, T263, W32

Cannabinoid ...................265, T278, W146

Cannabis . .18, M212, M250, M256, M262, M267, T54, W14, W146

Cannabis Dependence .................... T255

Cannabis Use . . 28, M33, M142, M182, M228, M253, W14, W262

Cannabis Use Disorder . . . . . . . . . . . . . . . . M260, T84, W252

Canonical Correlation Analysis (CCA) . . . . . . . . . . M96

Cardiac Autonomic Tone . . . . . . . . . . . . . . . . . M110

Cardiometabolic Risk .....................W151

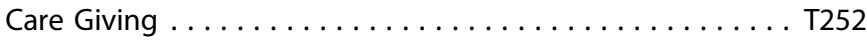

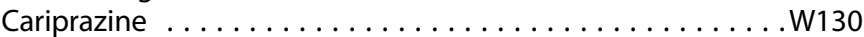

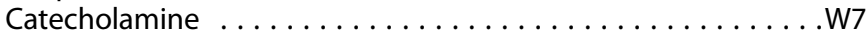

Caudate .......................... T243

Causal Reasoning .......................W165

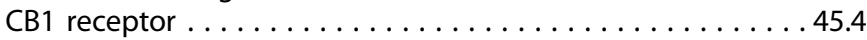

CBT ........................W10, W17

Cell Adhesion Molecule . . . . . . . . . . . . ...M274

Central Amygdala . . . . . . . . . . . . . . . . T12, T100, T264

Central Nervous System . . . . . . . . . . . . . . . . . . M157, W193

Central Nucleus of the Amygdala ..........M12, M19, T62

Cerebellum .......................W213, W223

Cerebral Blood Flow . . . . . . . . . . . . . . . . . . M42, W156

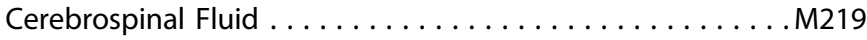

c-Fos . . . . . . . . . . . . . . . . . . . . . . . . . . . . M268

C-Fos-Expressing Ensembles . . . . . . . . . . . . T63, W232

Chemogenetics . . . . . . . 9.2, 45, T168, T278, W155, W231

Child Abuse and Neglect . . . . . . . . . . . . . . . T9

Child And Adolescent Psychiatry . . . . . . . . 33.4, 39.2, W129

Childhood Adversity . . . . . . . . . . . . . 47, M39, T275

Childhood Maltreatment . . . . . . . . . T9, T38, W40, W42

Childhood Trauma ........ . 54.3, M18, M107, T17, T89, T242,

T244, W57

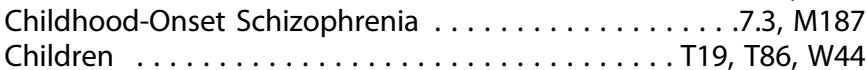

Children and Adolescents .........2.4, 54.3, M40, M199, T95,

W31, W34, W38

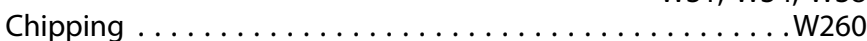

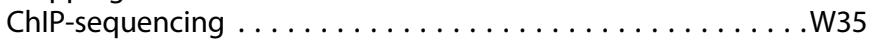

Choice Procedure . . . . . . . . . . . . . . . . . . M276, T267

Cholesterol Biosynthesis . . . . . . . . . . . . . . . . T246

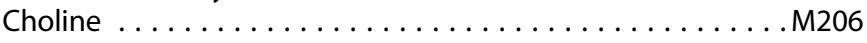

Cholinergic Anti-Inflammtory Response . . . . . . . . . . M112

Cholinergic System . . . . . . . . . . . . . M181, 768, W49, W71

Chromatin . . . . . . . . . . . . . . . . . . . . . . T14, W247

Chronic Mild Stress . . . . . . . . . . . . . . T103, T147, T151

Chronic Pain .......M150, T164, T165, T249, T254, W146, W250

Chronic Pain Treatment ...................31, W146

Chronic Social Defeat . . . . . . . . . . . . . . T219, T225

Chronic Stress . . . . . . 53.3, M260, T80, T274, T277, W87, W194

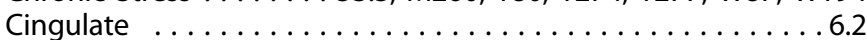

Cingulo-Opercular Network . . . . . . . . . . . . M20

Circadian . . . . . . . . . . . . . . . . . . . . . . . . W86, W282

Circadian Rhythm . . . . . . . . . . . . T52, T181, W82, W274
Circuit

$19,19.4, \mathrm{~T} 150$

Clinical High Risk

$29.1,29.2$

Clinical High Risk for Psychosis . . . . . . . 25.1, 29, 29.3, M133,

M195, M212, T194

Clinical High Risk State for Psychosis . . . 32, M182, M191, W215

Clinical Outcome Prediction ................W63

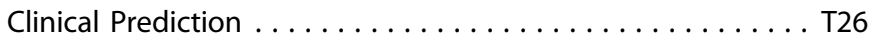

Clinical Predictors . . . . . . . . . . . . . . . . . . . . M59, T137

Clinical Psychopharmacology ................... M139

Clinical Stability ........................ M184

Clinical Subtypes ...................M202, W11, W166

Clinical Trial 12.1, 28, 32, M32, M246, T71, T119, T211, W31, W91

Clinical Trial Design . . . . . . . . . . . . . . . . T4

Clinical Trial Rating Methods ................W46

Clonidine ......................... T189

Clozapine ............................W220

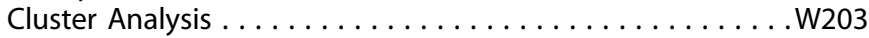

CNS Clinical Trials . . . . . . . . . . M94, T213, W74, W143

Cocaine ..........41.1, 45, 53.2, M233, M238, M246, M252,

M258, M272, T50, T60, T223, T232, T250, W97, W234

Cocaine Addiction ...........6.4, M274, T51, T235, T238,

T245, T257, W232, W235, W236, W242, W256,

W257, W260

Cocaine Reinstatement and Taking .......... T50, W233

Cocaine Seeking . . . . . . 41.3, 45.3, 45.4, M264, T268, W246

Cocaine Self-Administration . M278, T50, T52, T277, W230, W247

Cocaine Self-Administration and Reinstatement .T59, T226, T284

Cocaine-Related Disorders ... . . . . . . . . . . . T256, W264

Cognition .........33.2, 56, M49, M83, M133, M180, M190,

M195, M215, M236, M275, T41, T47, T58, T67, T166,

T185, T188, T193, T212, T238, T255, T281, W90, W147, W150, W211, W235, W281

cognitive appraisal . . .................. T75

Cognitive Behavior Therapy . . . . . . . . . . . . . . . T92

Cognitive Behavioral Therapy . . 2.1, M20, M87, M161, T164, T221

Cognitive Control . . . . . . . . . 2.2, M218, T191, W188, W205

Cognitive Control Network ... . . . . . . . . 2.1, M34, M79, T25

Cognitive Decline . . . . . . . . . . . . . . . 29.1, M53

Cognitive Enhancement . . . . . . . . . . . . . . . . . . . . 53.4

Cognitive Flexibility . . . . . . . . . . . . 15.2, W106

Cognitive Functioning $\ldots \ldots \ldots 40,54.2$, M82, T17, T71, W89

Cognitive Impairment . . . . . . . . . . . . . . . . . 29.3, . . . . . . . . . . . . . . T68, T178

Cognitive Neuroscience . . . . . . . . . . . . . M77, W60, W254

Cognitive Reappraisal . . . . . . . . . . . . . 46.1, 46.2

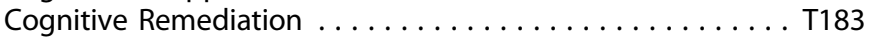

Cognitive Subtypes . . . . . . . . . . . . . . . . W125

Cognitive Training ...................... 29.4

Collaborative Cross and Diversity Outbred Mice ........ T52

Combat PTSD . . . . . . . . . . . . . . 3, 3.3, M19, M283, W172

Combat Veteran . . . . . . . . . . . . 3.2, 35.2, M160, T174

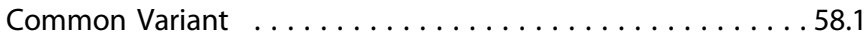

Comorbidity ..................T220, T244, W98, W215

Complement . . . . . . . . . . . . . . . . . . . . M69

Complement Component 4 . . . . . . . . . . . . . . . . . W197

Complement Factor $C 4 \ldots \ldots \ldots \ldots \ldots \ldots \ldots$. . . . . . 49.3

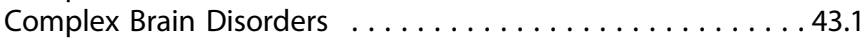

Compulsive Behavior . . . . . . . . . . . . . . . . . . 1.4

Compulsive Models Of Drug Use . . . . . . . M231, M233, M280

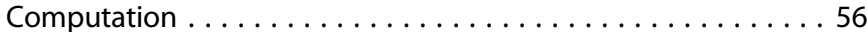

Computational Modeling .........3.3, 18, M15, M24, T120, W171

Computational Models Of Decision-Making . . . . . . . 3.2, T21

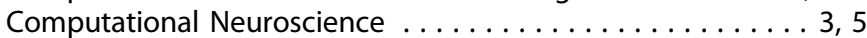

Computational Psychiatry .........3, 11.3, 18.1, 39, M37, M48,

T98, W171, W190

Computerized Cognitive Training ...... M171, M181, M190, T71

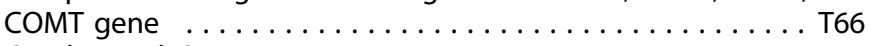

Conditioned Cues . . . . . . . . . . . . . . . . . . . . . . . . . . . W282

Conditioned Place Preference $\ldots \ldots \ldots \ldots \ldots \ldots \ldots \ldots$. . . . . . 
Conduct Problems . . . . . . . . . . . . . . . . . . . . . . 24.2

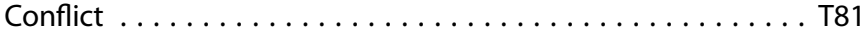

Conflict Monitoring ....................W17

Congenital Malformations $\ldots \ldots \ldots \ldots \ldots \ldots \ldots \ldots \ldots$ T145

Connection .............................. T93

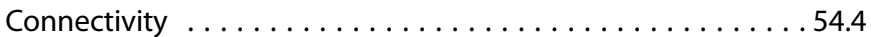

Connectivity-Based Parcellation .............. T153

Connectome . . . . . . . . . . . . . . . . . . . . 29.2, T257

Connectome-wide association study $\ldots \ldots \ldots \ldots \ldots$. . . . 43

Connectomics .............................. 158

Context . . . . . . . . . . . . . . . . . . . . . . . 50.4, W239

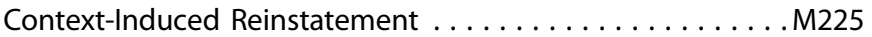

Contextual Fear . . . . . . . . . . . . . . . . . . . . . . . . M284

Copy Number Variant . . . . . . . . . . . . . . . . . 33.3, M41, W36

Copy Number Variation . . . . . . . . . . . . . . . . . . M42

Corpus Callosum . . . . . . . . . . . . . . . . . . . . . T172

Cortical Circuit Function . . 11.1, 25, M216, T39, T181, T182, T190,

Cortical Dysplasia . . . . . . . . . . . . . . . . . . T160

Cortical Excitation-Inhibition Balance ............. 36

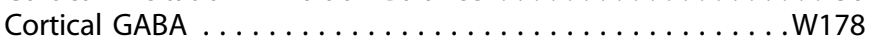

Cortical Layers ........................ T39

Cortical Plasticity . . . . . . . . . . . . . . . . . . T273

Cortical Thickness ...... . . 13.3, M35, M94, M200, M261, T242,

W18, W172

Cortical Thinning .....................W118

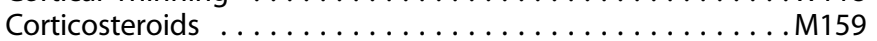

Corticosterone ..................T34, T96, T103

Corticostriatal Circuit . . . . . . . . . . . . . . . . . . . . T276

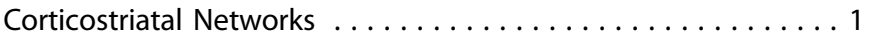

Corticotropin-Releasing Factor (CRF) . . . . . . . . 45.1, T231

Cortisol . . . . . . . . . . . . . M67, T8, T133, W118, W271

Craving .................T241, T247, W276, W280

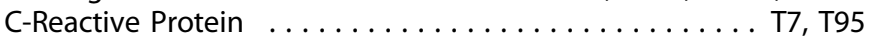

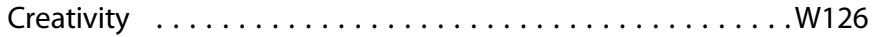

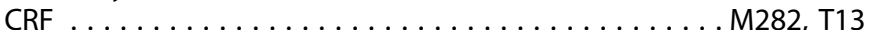

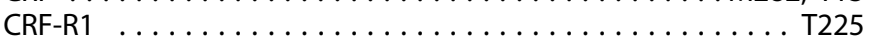

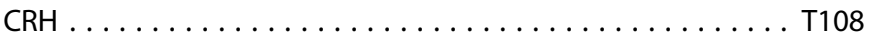

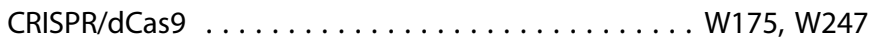

Cross Species ......................W78

CRP ......................M102, T1, T115, W138

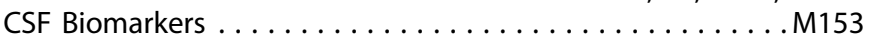

Cue Reactivity . . . . . . . . . . . . . M258, T55, T260

Cue Reinstatement . . . . . . . . . . . . . . . . . W245

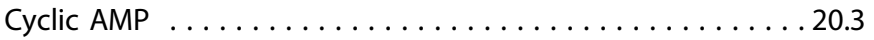

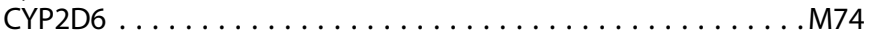

Cytokine ............. 26.3, M219, T105, T212, W236

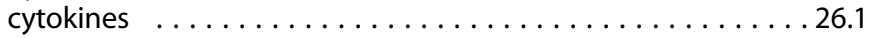

Cytomegalovirus ........................ T139

D1 Dopamine Receptors .........1.3, 1.4, M174, T231, W147,

W248, W270

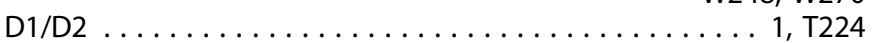

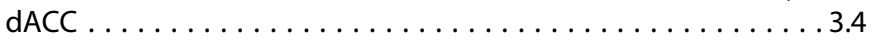

D-Amino Acid Oxidase ....................W187

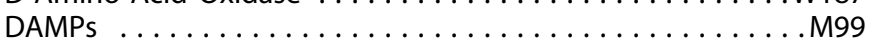

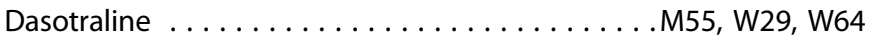

Data Discovery . . . . . . . . . . . . . . . . . . . . . . . . . . . . . . . . . . . . . . . .

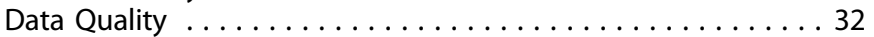

Data Sharing .......................... 138

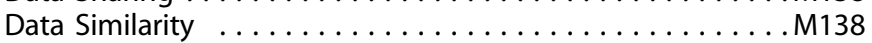

Database ..........................W183

d-cycloserine . . . . . . . . . . . . . . . . . . . . W134

Decision-Making . . . . . . . . . . . . . . . . 3.4, M67, M278

Deep Brain Stimulation . . . . . . . . . . M264, T37, W160

Deep Indexing . . . . . . . . . . . . . . . . . . . . . . . . M138

Default Mode Network (DMN) . . . . . . . . .M43, M189, T197

Delay Discounting . . . . . . . . . . . . . . . . . T241

Delirium ......................... 550
Delusions . . . . . . . . . . . . . . . . . . T204

Dementia .................. M236, T126, W51, W52

Dementia-Related Psychosis . . . . . . . . . . . . . . . . . W54

Dendritic Morphogenesis . . . . . . . . . . . . . . M175

Dendritic Remodeling . . . . . . . . . . . . . . . . . . . . 1.1

Dendritic Spine . . . . . . . . . . . . . . . . . . . . . . . . . . . 43.2

Dendritic Spines . . . . . . . . . . 1.1, 27.1, 40.4, W140

Dentate Gyrus . . . . . . . . . . . . . . . . . . . . . T2, T199, W228

Dependence ........................W255

Depression . . ............1, 1.2, 2, 2.4, 7.1, 8.4, 26, 26.3,

34.4, 35.3, 35.4, 42.2, 52.4, 53, 53.2, M53, M67,

M68, M74, M75, M76, M77, M78, M91, M92, M98, M105, M109,

M113, M120, M121, M124, M128, M129, M130, M161, M250, T8,

T25, T31, T34, T67, T84, T105, T110, T119, T125, T127, T128, T131,

T133, T135, T140, T142, T143, T146, T151, T190, T219, W13, W44,

W86, W94, W96, W98, W122, W135, W138

Depression Inflammation Cytokine ..... M99, T115, W76, W124

Depression Subtypes .................M115, T112

Depression-Like Behavior . . . . . . . . . M99, T33, T102, T103

Design ............................. M127

Development ....................54.4, T107, W215

Developmental Neuroscience . . . . . . . . . . . . . . . 39.2

Developmental Psychopathology . . . . . . . 39, M46, T17, T132

Developmental Trajectories . . . . . . . . . . . . . . 35

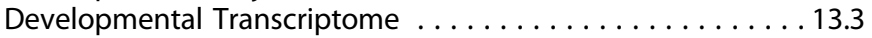

Deviance Detection ...................... M173

Dexmedetomidine ......................63

Dextromethadone ..................... T146

Diabetes ... . . . . . . . . . . . . . . . . . . . . . .W71

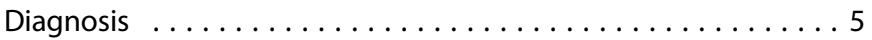

Diagnostic Boundaries . . . . . . . . . . . . . . . . 25.3, M220

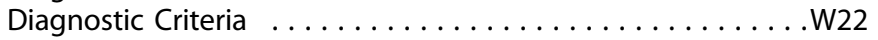

Diet Induced Obesity . . . . . . . . . . . . . . ...M46

Diffusion Tensor Imaging (DTI) . . 25.4, M4, M93, M192, M195, T9,

T177, W1, W62, W107

Diffusion Weighted Imaging . . . . . 54.2, M135, M210, T153, W75

Digital Assessment ................... 4.1, M82

Dimensional . ..................24.3, 39, 39.3

Dimensions . . . . . . . . . . . . . . . . . . . . 19.4

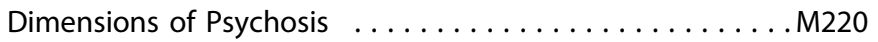

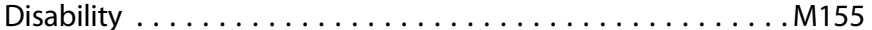

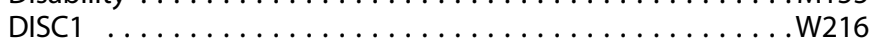

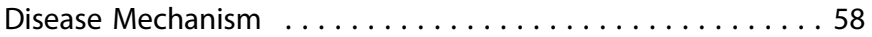

Disease Modeling . . . . . . . . . . . . . . . . . ..W81

Disorders of Glutamate . . . . . . . . 12.4, 27.3, W72, W198

Disrupted-in-Schizophrenia 1 . . . . . . . . . . . . . . . . T177

Disruptive Mood Dysregulation Disorder . . . . . . . . . . 39.2, M93

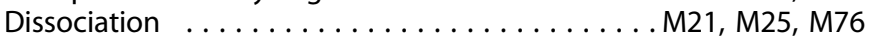

Diversity . . . . . . . . . . . . . . . . . . . . 22, M202, M224, T167

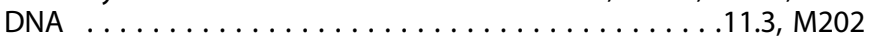

DNA Methylation .......M116, T7, T179, W16, W101, W249

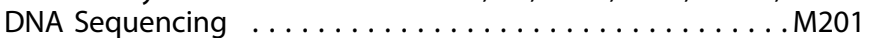

Dopamine . . . . . . . . . . . 20, 35, 35.3, 35.4, M64,

M122, M125, M126, M136, M211, M212, M247,

M282, T22, T64, T154, T163, T263, W4,

W69, W139, W149, W162, W168, W234, W242, W244, W264, W273,

W282, W284

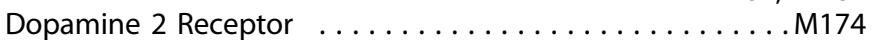

Dopamine Circuitry . . . . . . . . . . . . . . . . . .W70

Dopamine D2 Receptors ... . . . . . . . . . . . . . . T243

Dopamine D3 Receptors ............. 50, M235, T276

Dopamine Receptor Type 2-Expressing Striatal Medium Spiny Neuron

M264

Dopamine Receptors . . . . . . . . . . . . . . . . T37, W79

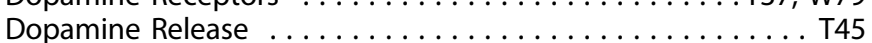

Dopamine Transporter . . . . . . . . . . . . . . T174, T268

Dopaminergic System . . . . . . . . . . . . . . . . . . T28, W90

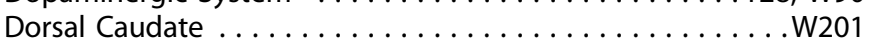


Dorsal Raphe Serotonin Neurons

M66, T80

Dorsal Striatum

$16.3, \mathrm{~T} 22, \mathrm{~T} 46$

Dorsolateral Prefrontal Cortex . . . . . . . . . . . . W62, W270

Dose Reduction .................... T43

Dose-Response Analyses .................. T35

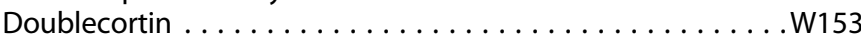

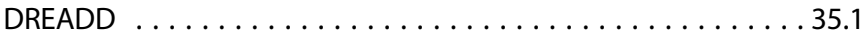

DREADD Receptor $\ldots \ldots \ldots \ldots \ldots \ldots \ldots \ldots \ldots$ T10

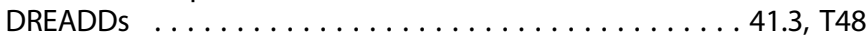

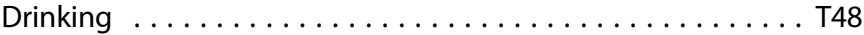

Drug addiction ......... 23, 23.4, T230, T282, W269, W274

Drug Cues . . . . . . . . . . . . . . . . . . . . . T250

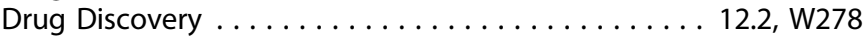

Drug Discovery - New Approaches . . . . . . . 7, 7.2, 31.2, W147

Drug Discovery/Development $\ldots \ldots \ldots \ldots \ldots \ldots 5$. M274

Drug Discrimination . . . . . . . . . . . . . . . . . . . . M269

Drug Relapse ..............16.1, 50.4, M268, T266

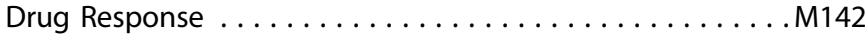

Drug Side Effects .................. M84, W192

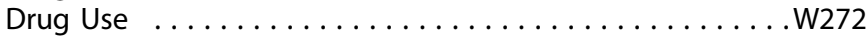

D-Serine .........................W187

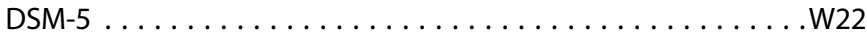

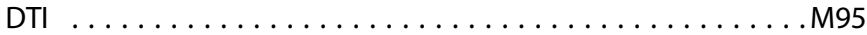

Dynamic Causal Modeling .................... M188

Dynamic Connectivity . . . . . . . . . . . . . . . . . . . . M248

Dynorphin . . . . . . . . . . . . . . T154, T157, T232

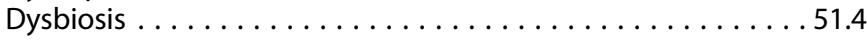

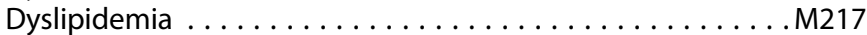

E/I Imbalance ........................ T109

Early Adolescence . . . . . . . . . . . . . . . . . . M17

Early Development . . . . . . . . . . . . . . . . . . . . . . . 54.1

Early Identification of Risk . . . . . . . . . . M37, M44, M227

Early Illness Schizophrenia ... . . . . . . . . . . M191, M193

Early Intervention . . . . . . . . . . . . . . . . . T71

Early Life Adversity . . . . . . . . . 51.1, M1, M39, M227, T108

Early Life Stress . . . . . . . . . . .37.1, 47, M5, M29, M260, T83,

T108, T139, T159, W30, W35, W41, W258

Early Phase Drug Development . . . . . . . . . 7.3, 12.1, 55 Early Psychosis . . . . . . . . . 32.1, M194, M200, M218, W207 Eating Disorders .................... M56, M59, W60 Ecological Momentary Assessment . . . . . . . . 4.2, W272 ECT . . . . . . . . . . . . . . . . . 19, 19.1, 19.4, M109, W83 EEG $\ldots \ldots \ldots \ldots \ldots \ldots \ldots \ldots . \ldots \ldots \ldots \ldots$ 10.2, M155, M156, T85, T178, W13, W48, W141, W226

EEG Biomarkers $\ldots \ldots \ldots \ldots \ldots \ldots \ldots \ldots \ldots \ldots \ldots$ T51

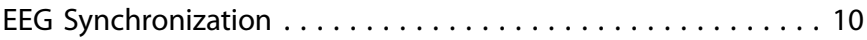
EEG/ERP Electrophysiology . . . . . . . . .T121, T189, W180, W191

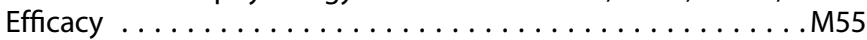

Effort Based Decision Making Task . . . . . . . . . . . . W65

Effort Choice Task . . . . . . . . . . . . . . . . . . T210

Effort Discounting . . . . . . . . . . . . . . . T201

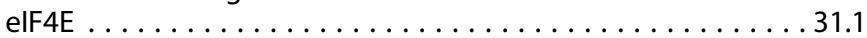

Electrical Field Modeling .............. M109, T153

Electroconvulsive Therapy . . 19.2, 19.3, M162, T28, T134, W120

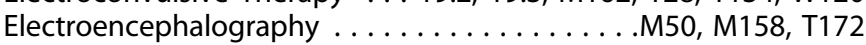

Electronic Health Record (Ehr) . . . . . . . . . . . . . T208

Electronic Medical Record ... . . . . . . . . . . . . . . . W73, W173

Electronic Prescribing Warnings . ..............M139 Electrophysiology .45.1, M126, M278, T12, T80, T191, W70, W140

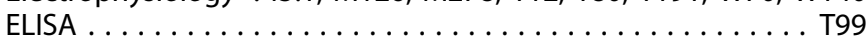

Emergency Medicine . . .................W21

Emotion . . . . . . . . . . . . . . . . 48.4, T89, T133, T255

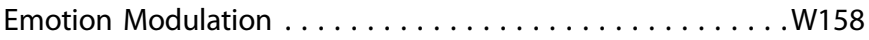

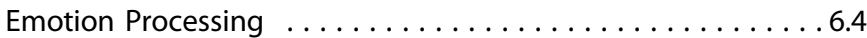

Emotion Recognition . . . . . . . . . . . . . . . . W 257

Emotion Regulation . . . . . . . . . 17.3, 46.2, M218, T18, T26

Emotional Processing . . . . . . . . . . . . . . . . . . . . W103

Emotional Regulation ..............M171, W108, W119
Emotional Response Inhibition . .............. M45

Emotional Stress . . ...................M28

Empathy . . . . . . . . . . . . . . . 9, 9.2, 9.3, W126

Endocannabinoid System . . . . . . . . . . . . . 32, M169, M260

Endocannabinoids ........31.3, 32.1, M167, T79, T163, T277

Endophenotypes . . . . . . . . . . . . . . . . . 7, M220

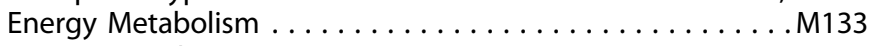

ENIGMA Working Group . . . . . . . . . . . . . . . . . . . . . . . T104

Enkephalin . ..................... T157

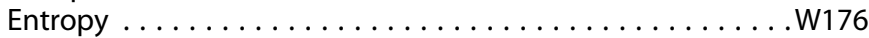

Environment .......................W260

Environmental Risk Factors . . . . . . . . . . . . . W259

Epidemiology . . . . . . . . . . . . . . . . . . . . M137

Epigenetic Modification . . . . . . . . . . . M236, M244, W101

Epigenetics ....... 13, M107, M172, M240, T65, T179, T233,

T234, T264, W16, W35, W42, W67, W78, W242

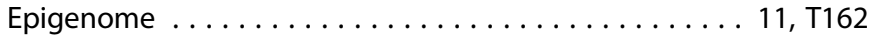

Epigenomics ........................ 52

Epilepsy ..............27.1, M130, T160, W141, W167

Epiodic Memory . . ................... T66

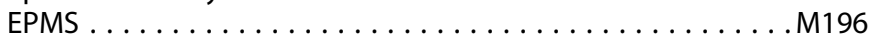

eQTL $\ldots \ldots \ldots \ldots \ldots \ldots \ldots \ldots \ldots \ldots \ldots \ldots \ldots$ 44.4, T207

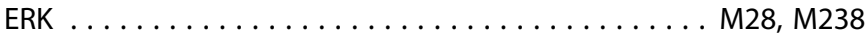

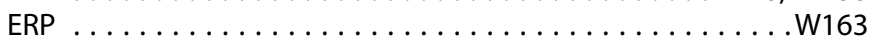

Error Processing ................... M209, W163

Escitalopram .................. M2, M74, M91

Esketamine . . . . . . . . . . 42.1, M76, M269, T125, W131

Estradiol . . . . . . . . . . . M97, M108, M125, T66, T74

Estrogen Receptor . . . . . . . . . . . . . . . . . . . . . T185

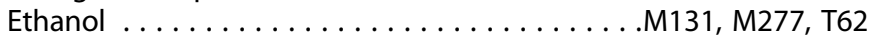

Ethical Issues . . . . . . . . . . . . . . . . . . . . . . . . . M137

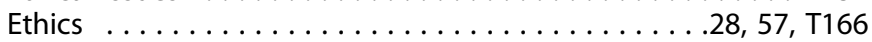

Event-Related Potentials . . . . . . . . . . . . M35, T194

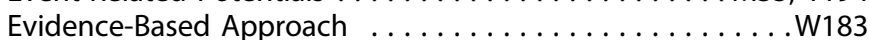

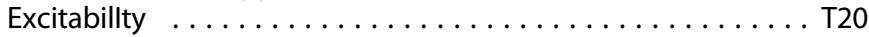

Excitatory Synapses $\ldots \ldots \ldots \ldots \ldots \ldots \ldots \ldots \ldots \ldots \ldots \ldots \ldots \ldots \ldots \ldots \ldots \ldots \ldots \ldots \ldots \ldots .1,27.2$

Executive Function $\ldots \ldots \ldots \ldots \ldots \ldots \ldots \ldots \ldots \ldots \ldots \ldots$. . . . . . . .

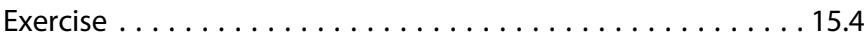

Exosomes . . . . . . . . . . . . . . . . . . . . . . . . . . . M237

Exosomes of Neuronal Origin . . . . . . . . . . . . . M197

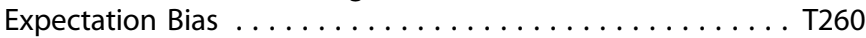

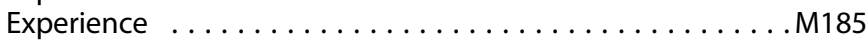

Experimental Medicine . . . . . . . . . . . . . . . 12.1

Experimental Therapeutics ............12.4, M79, T156

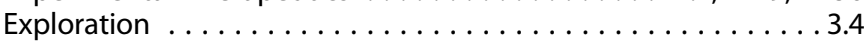

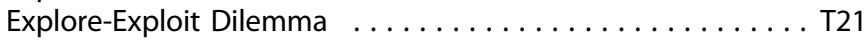

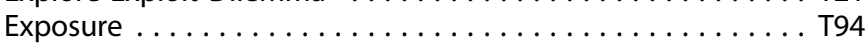

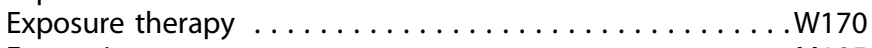

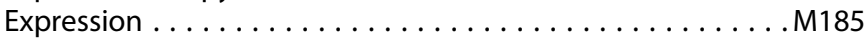

Extended Amygdala $\ldots \ldots \ldots \ldots \ldots \ldots \ldots \ldots \ldots \ldots$. $\ldots \ldots \ldots$

Extended-Release Depot . . . . . . . . . . . . . . M184

Extended-Release Naltrexone . . . . . . . . M245, T248, W250, W253

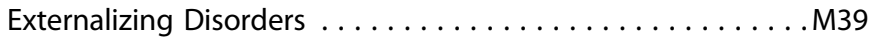

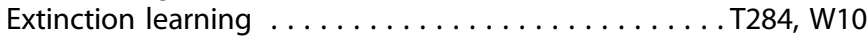

Extinction Memory Recall ... . . . . . . . . . . . . T5, W171

Extracellular Matrix . . . . . . . . . . . 49, 49.4, T49, W87

Extracellular Vesicles . . . . . . . . . . . . . . . . . . . . . . . . . M237

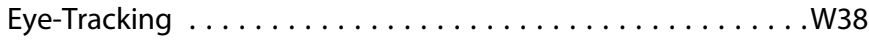

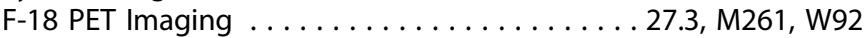

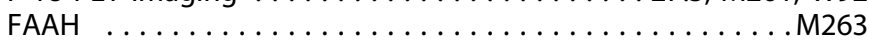

Face Emotion Processing $\ldots \ldots \ldots \ldots \ldots \ldots \ldots \ldots$. . . . . . . . . . .

Facial Emotion Processing ... . . . . . . . . . . M191, W24

Factor Analysis ......................W213

Fast Scan Cyclic Voltammetry . . . . . . . . . . . W273

Fast-Acting Antidepressant . . . . . . . . . . . . . . W133

Fatty Acid Amide Hydrolase . . . . . . . . . . . . . . . M169

Fatty Acids . . . . . . . . . . . . . . . . . . . . . . . . . . . M49

Fear .................... T12, W6, W18 
Fear Conditioning 1.2, 48.2, 48.4, M5, M7, T70, T75, T79, T107, T173, W226

Fear Extinction .................................... M167, T5, W8

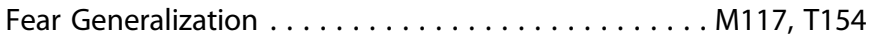

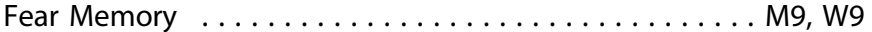

Feed Forward Inhibition . . . . . . . . . . . . . . . . . . . . T270

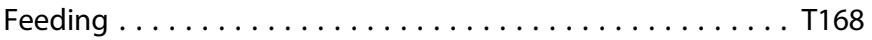

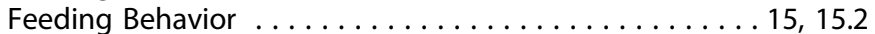

Female . . . . . . . . . . . . . 42.2, T169, T225, W28, W97

Fentanyl . . . ..................... M232, T267

Fetal Alcohol Syndrome . . . . . . . . . . . . . . . . . . . . . . M244

Fetal Brain Development . . . . . . . . . . . . . . 58.4

Fetal Programming . . . . . . . . . . . . . . . . .W57

First Episode Psychosis . . . . . . M221, T186, W201, W213, W227

First Episode Schizophrenia . . . . . . . . . . . . M187, T209

First-Episode Psychosis . . . . . . . . . . . . . . . . . . . . . . T208

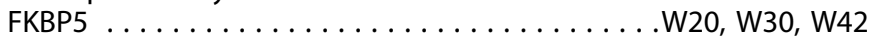

Flanker Task ........................ T121

Fluorodeoxyglucose ...............M160, M211

Fluoxetine ... . . . . . . . . . . . . . . . 1.4, T34, W28, W97

fMRI Biomarkers . . . . . . . . . . . . 12.4, 25.1, M27, M88

fMRI Effective Connectivity . . . . . . . . . . . . . . T91

fMRI Functional Connectivity . . . . . . . . . 29.3, M257, W104

fMRI Negative Affective Stimuli . . . . . . . . . . . . W57

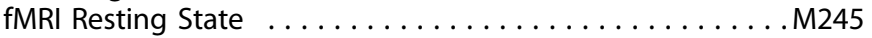

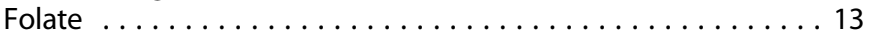

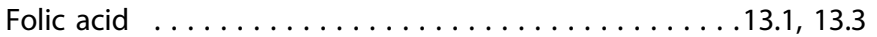

Food Addiction . . . . . . . . . . . . . T97, T285, W232

Food Intake . . . . . . . . . . . . . . . . . . . . . . 15.4

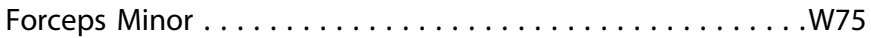

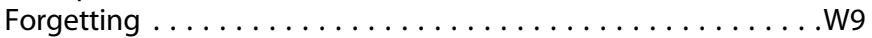

Fractional Anisotropy . ................W56

Fragile X Syndrome .............. M146, M149, T85, W32

Frontomedial Negativity . . . . . . . . . . . . . . . . . . M193

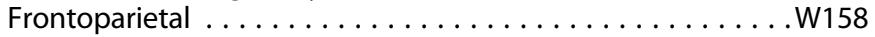

Frontostriatal Circuitry .................... M135

Frustration ........................W106

Frustration Tolerance $\ldots \ldots \ldots \ldots \ldots \ldots \ldots \ldots \ldots \ldots$ T18

FTO ... . . . . . . . . . . . . . . . . . . . . . . . . . . T19

Functional and Structural MRI ...............W167

Functional Capacity ........................ 25

Functional Characterization ................ 58

Functional Connectivity . . . . . . 24.1, M100, M142, T42, W202

Functional Genomics . . . . . . . . . . . . . . . . 11.3, 11.4

Functional MRI (fMRI) . . . . . . . 2.2, 3.2, 12.1, 12.3, 15.4, 18.1,

29.2, 35.3, 40.3, 41.2, 46.1, 46.2, 48, 48.4, 56.3, M16, M34, M44, M45, M56, M57, M79, M91,

M96, M142, M161, M165, M188, M189, M207, M209, M249, M252, M256, M258, M260, M262, T16, T36, T37, T54,

T55, T59, T66, T69, T72, T129, T135, T175, T190, T201,

T221, T240, T248, T250, T252, T255, T258, T260, W5, W17, W19, W34, W38, W61, W62, W63, W99, W103, W106, W108, W109, W119, W176, W205, W218, W256, W266, W269, W275

Functional Neuroimaging . . . . . . . . . M17, T88, T89 G Protein . . . . . . . . . . . . . . . . . . . . . . 20.3

G Protein Coupled Receptors . . . . . . . . . . . . 43.3

G Protein Signaling . . . . . . . . . . . . . . . . . . . . 43.3, M239

G Protein-Coupled Receptors . . . . . . . . . . . . 7.2

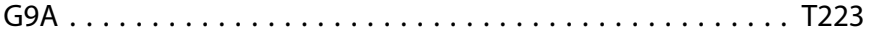

GABA . . . 8 8. 8.2, 8.4, M65, M162, T14, T15, T48, T109, T148,

T161, W135

GABA Transmission . . . . . . . . . . . . . . . . . . . W279

GABA-A Receptor Positive Allosteric Modulator . . . 8.3, 8.4, W148 GABA-A Receptors . . . . . . . . . . M215, T161, T182, W140

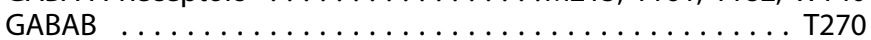

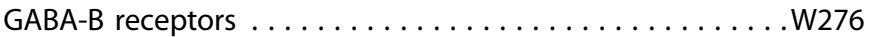
GABAergic Interneurons . . . . . . . . . . . . . . 10.4, M216, M223

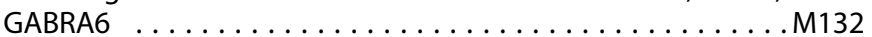

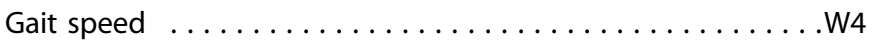

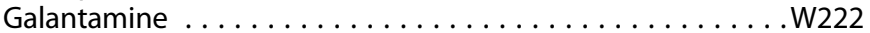

Gambling Disorder . . . . . . . . . . . . . . . . . . . T239, T251

Gamma Oscillation . . . . . . . . . .34, 34.4, 36, T163, T217

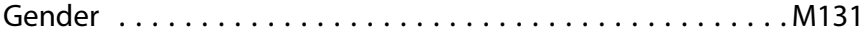

Gender Differences . . . . . . . . . . . . . . . . . . 42

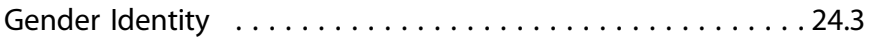

Gene Environment Interaction . . . . . . . . . . . . . . . . W41

Gene Expression ..44.3, M12, M13, M131, T60, T112, T181, W231

Gene Transcription . . . . . . . . . . . . . . . T179, W175

Genecept Assay . . . . . . . . . . . . . . . . . . . . . . . . . . . . M147

Genetic Animal Model . . . . . . . . . . . . . . . . . .W231

Genetic Association ...................... 58

Genetic Association Study ... . . . . . . . . . . . . M187, W197

Genetic Liability . . . . . . . . . . . . . . . . . . . . T191

Genetic Mouse Model . . . . . . . . . . . . . . . . . . . . W178

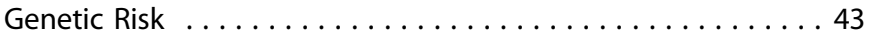

Genetic Score ...........................W37

Genetic Testing . . . . . . . . . . . . . . . . . . . 57

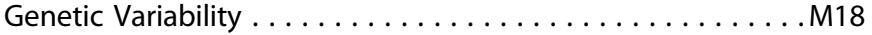

Genetic Variation . . . . . . . . . . . . . . . . . . W216

Genetics .............................M201

Genome-Wide Association Study . . . . . . . 44, 44.1, 44.4, M201

Genomic . . . . . . . . . . . . . . . . . . . 33, 52.4

Geriatric .......................... T69

Geriatric Depression . . . . . . . . . . . . . . . M2, W95

Ghrelin ..................... 15.3, W271

Glia .................................26.1

Global Assessment of Functioning (GAF) . . . . . . . . . T114

Globus Pallidus ...................... T42

GLT-1 . . . . . . . . . . . . . . . . . . . . . . . T279, W245

Glucocorticoid Dysregulation . . . . . . . . . . 23.4, W30

Glucocorticoid Receptor . . . . . . . . . . . . 23.4, M70, W249

Glucocorticoids . . . . . . . . . . . . . . . . . . M1, T277

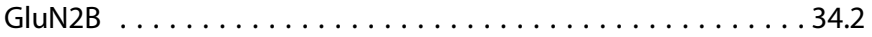

GluN2C subunit .....................W223

Glutamate . . . . . 12.2, 20.2, 49, M1, M71, M90, M136, M234,

T106, T148, T215, W55, W201, W245

Glutamate GABA . . . . . . . . . . . . . . 34, 34.2, T127, T205

Glutamate Homeostasis . . . . . . . . . . . . . . T279, T281

Glutamate Receptor Function . . . . . . . . . . . . 20, 27, 43.4

Glutamatergic Synapses . . . . . . . . . . . . . . . . . . . . 20.2

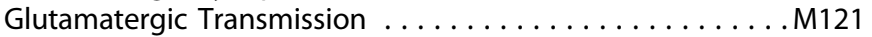

Glutamine . . . . . . . . . . . . . . . . . . . . . . . . . . . T127

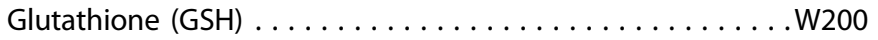

Goal-Directed Behaviors .......................W239

Goal-Directed Control . . . . . . . . . . . . . . . . . . . . . . . . . . . T261

Gonadal Hormones . . . . . . . . . . . . . . . . . M9, T23

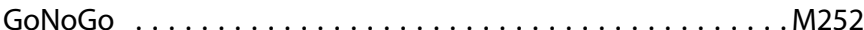

GPCR . . . . . . . . . . . . . . . . . . . . . 20, 20.3, W278

Graph Theory . ................... 19.2, T101

Graph-Based Analysis . . . . . . . . . . . . . . . . . . . . W176

Gray Matter Volumes . . . . . . . . . . . . . . . . . T242

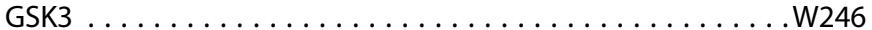

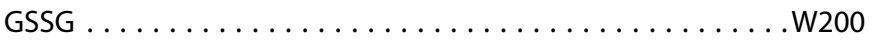

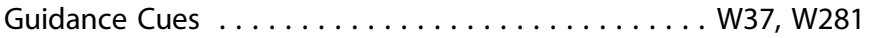

Gut Microbiome . . . . . . . . . . . 51, 51.1, M38, T105, T221

Gut-Brain Axis . . . . . . . . . . . . . . . . . . 51.4, T221

GWAS . . . . . . . . . 44.1, 58.2, T53, T162, T166, T199, W195

GWAS LOCI . . . . . . . . . . . . . . . . . . . . . . M274

habenula ....................... M101, M249, W104

habit . . . . . . . . . . . . . . . . M67, T22, T237

habit formation .................... T64

habits .................... 6.2, W60

habituation . . . . . . . . . . . . . . . . . . . . . M221

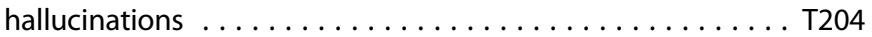

Hallucinogen . . . . . . . . . . . . . . . . . . . . . . . M226, T222

HCN .......................... 123 


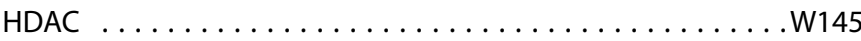

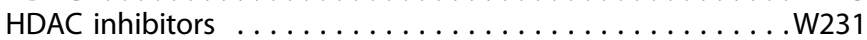

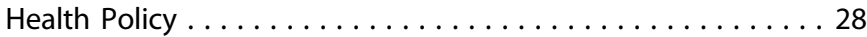

Health-Related Quality Of Life $\ldots \ldots \ldots \ldots \ldots \ldots \ldots \ldots .4$

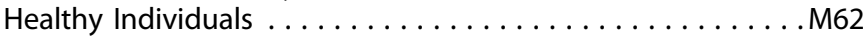

Healthy Subjects . . . . . . . . . . . . . . . . . . . M255

Heart Rate Variability . . . . . . . . . . . . M171, T241

Heat Shock Protein $70 \ldots \ldots \ldots \ldots \ldots \ldots$. . . . . . . . . . . . . . .

Hemodynamic Response Function . . . . . . . . . . . M165

Heroin Self-Administration . . . . . . . . . . . T49, T224, T282

Herpesvirus . . . . . . . . . . . . . . . . . . . . . . . . T139

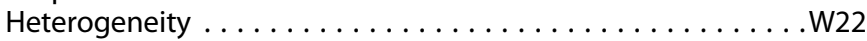

Hierarchical Clustering . . . . . . . . . . . . . . . .56.3, M115

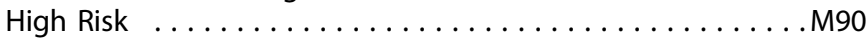

High Throughput Screening $\ldots \ldots \ldots \ldots \ldots \ldots \ldots \ldots \ldots .4$

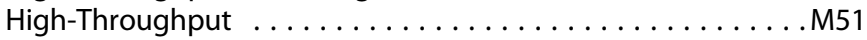

Hippocampal Shape ....................W118 Hippocampal subfields . . . M159, T131, T253, W39, W120, W182 Hippocampal Volume . . . . . . . . . . . . . . . . . . . M92 Hippocampal-Prefrontal . . . . . . . . . . . . . . . . . . M223 Hippocampus . . . . . 1.2, 16.2, 19.1, 27.2, 45.4, M68, M72, M98, M121, M129, M163, M167, M253, T104, T110, T186, T195, T199, T253, W228

Hippocampus-mPFC Pathway . . . . . . . . . . . . . . . 40.1 hiPSC $\ldots \ldots \ldots \ldots \ldots \ldots \ldots \ldots \ldots \ldots 44,58,58.2$, W81

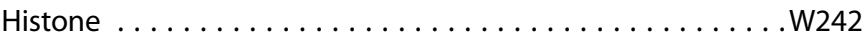
Histone Deacetylase . . . . . . . . . . . . . . . . . . . T224

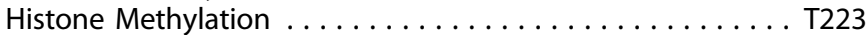
HIV ......................... T159, W251 HIV-Associated Neurocognitive Disorder . . . . . . . M230, M241 Hoarding Disorder ...................W11 Homeostasis ......................... 10 Homeostatic Plasticity . . . . . . . . . . . . . . 34.4 HPA Axis . . . . 23.4, 37.1, 52.3, M70, T8, W20, W41, W67, W121

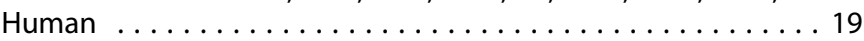
Human Brain . . . . . . . . . . . . . . . . . . . 11 Human Brain Imaging . . . . . . . . . . . . . 18, W263 Human Clinical Trial .................... M181, W113 Human Connectome ...................... T153 Human Genetics 33, 57, 58.3, 58.4, M201, T53, T167, W15, W173, W218

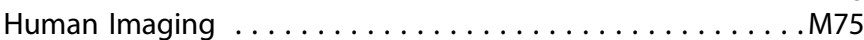
Human Laboratory Study . . . . . . . . . . . . . . . T79 Human Neuroimaging . . . . . 24, 29, 33, 35.4, 46, 46.3, M105, M250, T128, T251, W172, W268

Huntington's Disease . . . . . . . . . . . . W74, W145 Hydroxynorketamine ..... 20.1, 42.1, 42.2, M121, M127, T31, W131, W133, W135

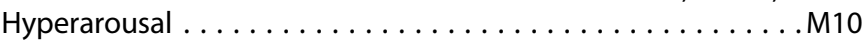
Hypersexual Disorder . . . . . . . . . . . . . . . .W67

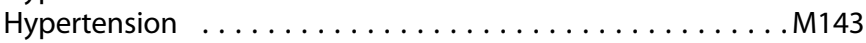
Hypobaric hypoxia . . . . . . . . . . . . . . . . . W259

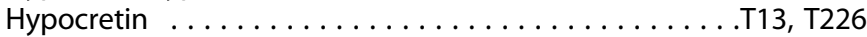
Hypothalamus ........... M6, M166, M244, T108, T180

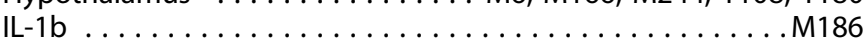

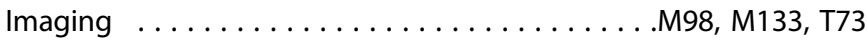
Imaging Genetics . . . . . . . . . . . . . . . . . . T249 Immediately Early Gene . . . . . . . . . . . . . . . M73 Immobility . . . . . . . . . . . . . . . . . . . T96 Immune . . . . . . . . . . . . . . . . . . . . . 35, W197

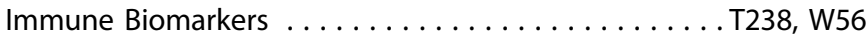
Immune Dysfunction . . . . . . . . . . . . . . . . . . . . . T139, W261 immune Markers . . . . . . . . . . . . . . . . . 26.1 Immune Responses ...................W216 Immune System . . . . . . . . . . . . . . . . . . . . . M157, M275 Immunopharmacotherapy $\ldots \ldots \ldots \ldots \ldots \ldots \ldots \ldots .2$ Impulse Control . . . . . . . . . . . . . . . . . . . .6.3

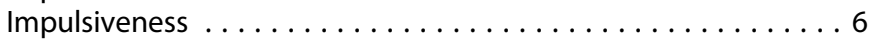

Impulsivity 30, 39.4, M62, M64, M65, M254,

T56, T261, W69

In Vitro Neuronal Differentiation .M146 In Vivo ............................W148 In Vivo Calcium Imaging . . . . . . . .15.1, 16.2, 16.4, 40.2, 40.4, M10, M280, T2, T46, T63, T272, W27, W228

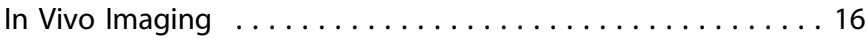
In Vivo Microscopy ........................ . 1.4 Incubation of Cocaine Craving . . . . . . . . . . . . . T274 Incubation of Craving for Cocaine And Nicotine . . . . . . . M229 Incubation of Drug Craving ... . . . . . . . . . . . . M272, T51 Individual Differences . . . . . . . . . . . . . . . . M231 Induced Pluripotent Stem Cells (iPSCs) 7, 7.3, 44.3, 58.1, 58.3, 58.4, W192, W196, W212

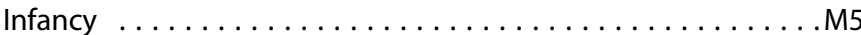
infant .................... 39.1, 54, W56, W57 Inference and Learning $\ldots \ldots \ldots \ldots \ldots \ldots \ldots \ldots \ldots$ W171 inflammation .......35.3, M83, M89, M113, M150, M186, T1, T32, T47, T115, T140, T212, T254, W7, W42, W138

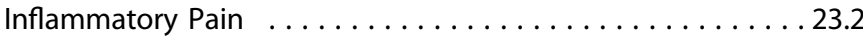

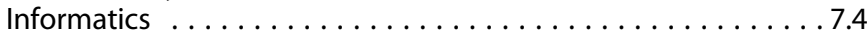

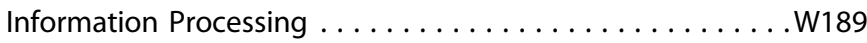
Infralimbic Cortex . . . . . . . . . . . . . . . . . . . T106

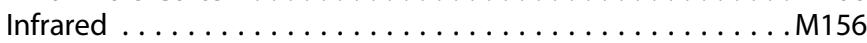

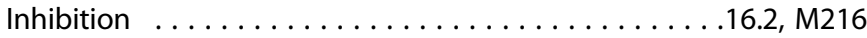

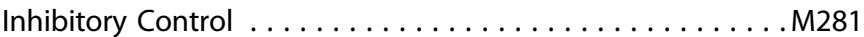

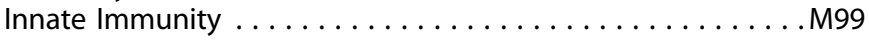

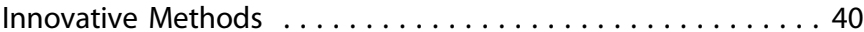

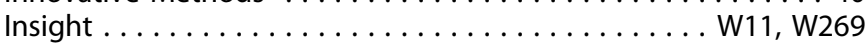
Insula . . . . . . . . . . . 9.2, 15.1, M57, T129, W57

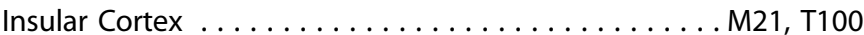
Insulin .......................... M106, T180 Insulin Resistance .................... M107, W193 Interactomes . . . . . . . . . . . . . . . . . . .44.1

Intergenerational Transmission . . . . . . . . . . 48, 48.3 Interleaved TMS/fMRI . . . . . . . . . . . . . . . . . M80, W158

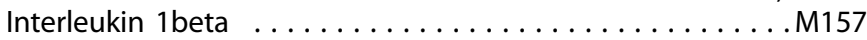

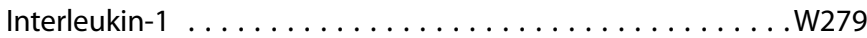
Internalizing Disorders . . . . . . . . . . . . . . . . . . . M39

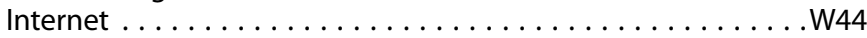
Interneurons ........................ 40.1 Interoception .............6.4, M23, M59, T129, W94

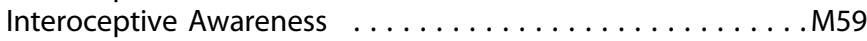

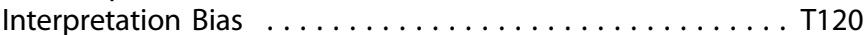
Intestinal Microbiome ....................W124

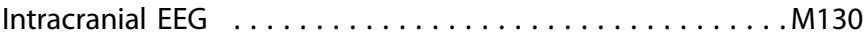
Intracranial Self-Administration . . . . . . . . . . . . . . . . . T264 Intracranial Self-Stimulation .................. M66 Intranasal Esketamine . . . . . . . . . . . . . T116, T118, W89

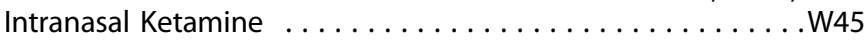
Intravenous Drug Self-Administration . . . . . . M235, T233

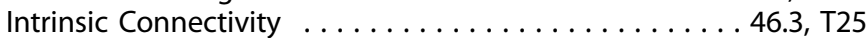
Intrinsic Functional Connectivity $\ldots \ldots \ldots \ldots \ldots \ldots$ T36 Ion Channels ..................... 7.1, M51 Irritability ............ M93, T18, W38, W74, W106

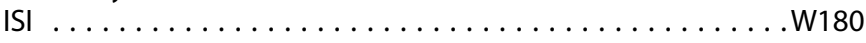
Journal Function . . . . . . . . . . . . . . . . . . . . . . . W164

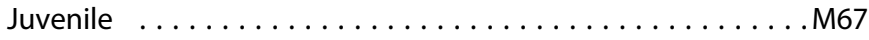
Kainate Receptor . . . . . . . . . . . . . . . . . . . . . T70 Kappa Opioid Receptor ....23.2, T147, T154, T232, W136, W277 Kappa Opioid Receptor Antagonist . . . . . 12, 12.3, T187, W168 Kappa Opioid Receptors . . . . . . . . . . . . . . . . M140, T268 Ketamine $\ldots \ldots \ldots \ldots \ldots \ldots . \ldots .1,20.2,34,34.2,40.4,42$,

42.1, 42.2, M32, M71, M76, M77, M106, M117, M120, M121, M122, M123, M127, T31, T32, T119, T125, T143, T144, T148, W10, W23, W45, W96, W103, W104, W109, W127, W133, W135, W137, W223 
Kinase .

Kinome

Knock-in mouse

Kynurenic Acid

Kynurenine

Kynurenine Pathway $\ldots \ldots \ldots \ldots \ldots \ldots \ldots \ldots \ldots \ldots \ldots$ T218

Language .............................. M189

Large Scale Networks . . . . . . . . . . . . . . . . M245, W159

Late-Life Depression . . . . . . . M94, M108, M112, W1, W4, W150

Latent Class Analysis . . . . . . . . . . . . . . . .W254

Lateral Habenula . . . . . . . . . . . . . . . . . . . . . . . . . . 34, M28

Lateral Hypothalamus . . . . . . . . . . . . . . . . T13, T168

Learning . . . . . . . . . . . . . . . . . . . . .W.W162

Learning Disability ......................... . . . . . . . . . . . . .

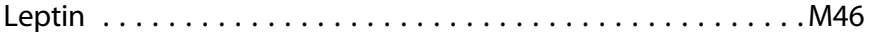

Lethality Studies . . . . . . . . . . . . . . . . . 50.2

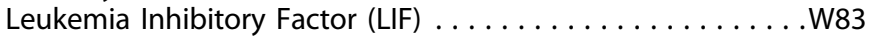

Levomilnacipran ...........................994

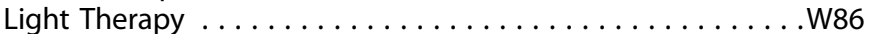

Likelihood to be Helped or Harmed . . . . . . . . . W186

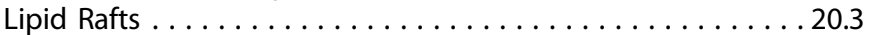

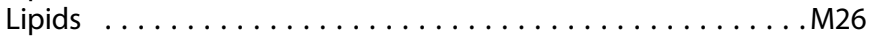

Lisdexamfetamine . . . . . . . . . . . . . . . . . . . .W64

Lithium . . . . . . . . . . . . . . . . . . .T122, T124, W82, W129

Lithium Response .................. T109, W111

Lithium Treatment . . . . . . . . . . . . . . . . . . . . . . . . M104

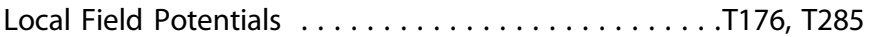

Locomotor Activity . . . . . . . . . . . . . . . . . . . . . . . . M240

Locus Coeruleus . . . . . . . . . . . . . . . . . . . . . . . . M63, M222

Long Access Self-Administration . . . . . . . . . 23.3, W248

Long Non-Coding RNAs . . . . . . . . . . . . . . . T110

Long Term Depression . . . . . . . . . . . . . . . . . . T284

Long Term Potentiation . . . . . . . . . M167, T156, T173

Longitudinal . . . . . . . . . . . . . . . . . 46.2, W16

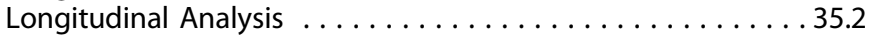

longitudinal imaging ............ 39.3, T131, W34, W39

Longitudinal Multimodal Imaging .................W179

Longitudinal Study . . . . . . . . . . . . T51, T114, W260

Long-Term Safety . . . . . . . . . . . . . . . . . . . . . . . T184

Long-Term Treatment . . . . . . . . . . . . . . . . . . T124

Long-Term Treatment of MDD . . . . . . . . . . . . . . . T141

LSD . . . . . . . . . . . . . . . . . . . . . . . . . . T80, T222

Lurasidone ..................... T95, W134

M1 and M4 Muscarinic Receptors ............ T45, W51

Macaque Monkey . . . . . . . . . . . . . . . . . . . . M173

Machine Learning . . . . . . . . . . . . . 11.4, 19.2, 39.3, 54.2, M27,

T98, T129, T162, T175, W73, W117, W125, W173

Machine Learning Classification M4, M93, M101, T208, W40, W202

Machine Learning Clustering . . . . . . . . . . . . . . . . . . M48

MADRS ..........................W85

Magnetic Resonance Imaging .... T19, T130, W31, W107, W201

Magnetic Resonance Spectroscopy . . . . . . . . . . . . . . . . M197

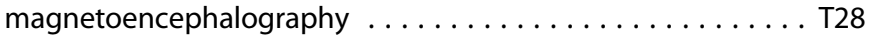

Maintanance ......................... 38

Major Depression ........... 46, M123, T130, W95, W118

Major Depressive Disorder (MDD) . . . . 8.4, 15, 15.3, 19.1, 19.2, M14, M79, M80, M84, M87, M88, M100, M102, M103, M110,

T32, T104, T106, T113, T118, T123, T138, T139, T144, T249,

W5, W85, W87, W103, W104, W107, W109, W125, W127, W252

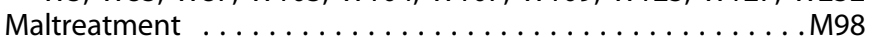

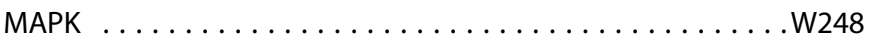

Marijuana . . . . . . . . . . . . . . . . M262, T263, W49

Marijuana Policy . . . . . . . . . . . . . . . . . . . . . T263

Mass Spectrometry . . . . . . . . . . . . . . . . . . M205

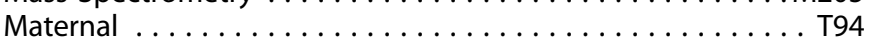

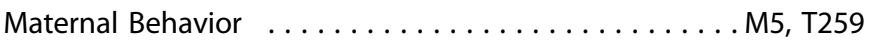

Maternal Depression . . . . . . . . . . . . . . . . . . . . . . . . . . 39.1

Maternal Immune Activation ......... 39.1, M30, M60, T176
Matrix Metalloproteinase-9 (MMP-9) . . . . . . . . . . . . . . T49

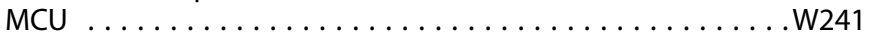

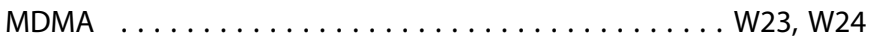

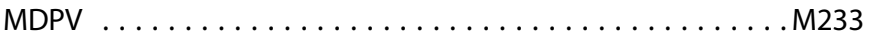

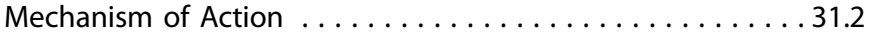

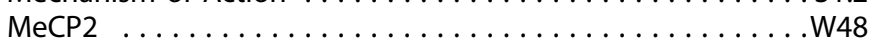

Medial Amygdala ................... T60

Medial Forebrain Bundle ..................W160

Medial Geniculate Nucleus . . . . . . . . . . . . . . . . . . . T284

Medial Prefrontal Cortex . . . . . . . . 34.2, 40.2, 40.4, M21, M174, M213, M280, W27, W50, W69, W84, W87, W277, W279

Medial Septum . ....................M129, W70

Medication Adherence ... . . . . . . . . . . . . . . . . M208

Medication Assisted Treatment . ....................... 266

Mediodorsal Nucleus of the Thalamus . . . . . . . . . . . . . 49.4

Medium Spiny Neuron . . . . . . . . . . . . . . . . . . 1.1, M119, T49

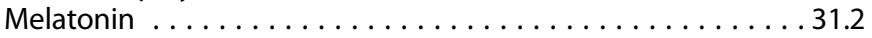

Memantine ........................... M2, M159

Memory ................ M159, M262, T72, W162

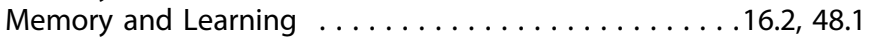

Memory Encoding and Retrieval . . . . . . . . . . 45, M250

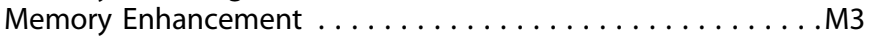

Memory Function . . . . . . . . . . . . . . . . . M1, M197

Memory Reconsolidation ...........45, 45.4, W9, W246

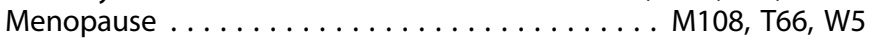

Mental Health . . . . . . . . . . . . . . . . . . . . . 24.3

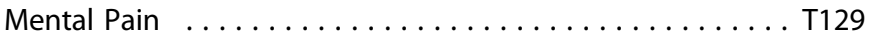

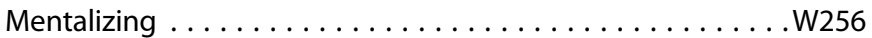

Mesocorticolimbic . . . . . . . . . . . . . . . . . . . T33

Mesolimbic Circuitry . . . . . . . . . . . . . . . . . M58

Mesolimbic Reward Circuitry . . . . . . . . . . . . . . M228, M277

Meta-Analysis . ................M44, T16, T187, W222

Metabolic Side-Effects . . . . . . . . . . . . W199, W207

Metabolic Syndrome . . . . . . . . . . . . . . . . . T7, W225

Metabolism ........................W263

Metabolites .........................M120

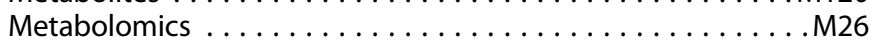

Metabotropic Glutamate Receptor . . . . . . . . . . . W262

Metabotropic Glutamate Receptor 2 (mGluR2) . . . . M107, T280,

Metabotropic Glutamate Receptor 3 (mGluR3) ......W221

Metabotropic Glutamate Receptor 5 (mglu5) . . . . . . . . . T59

Metalloproteases ......................449.4

Methamphetamine .............. M245, M255, W24, W270

Methodologies .................... T211

Methylation ....................... 13

Methylphenidate ................W29, W65, W157

Mexican Americans . . . . . . . . . . . . . . . . T123

mGlu $2 / 3$ Receptor ........................ T31

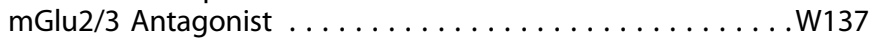

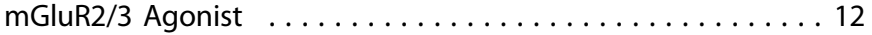

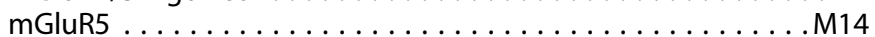

mGluR5 Receptors ................ 49.2

Mice ........................ T82

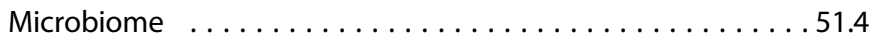

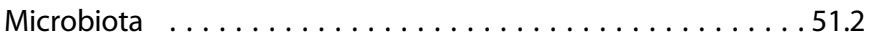

Microbiota-Gut-Brain Axis . . . . . . . . . . . . . . . . . . . . 51.1

Microdialysis . . . . . . . . . . . . .W47, W69, W131

Microglia . . . . . . . . . . 26, 49.3, M69, T44, T108, W1, W92

Microglial Activation . . . . . . . . . . . . . . . . . . . T254

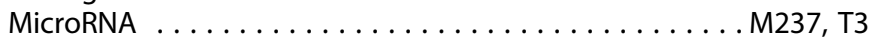

Midline Theta Frequency . . . . . . . . . . . . . . . . . . . T191

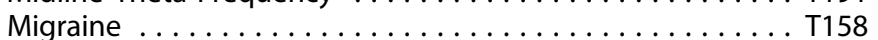

Mild Traumatic Brain Injury . . . . . . . . . . . . . M160

Mind \& Body Approaches . . . . . . . . . . . . . . . . . . . T220

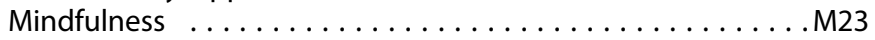

Miniscope . . . . . . . . . . . . . . . . . . . . . . W283

Minocycline $\ldots \ldots \ldots \ldots \ldots \ldots \ldots \ldots \ldots \ldots \ldots \ldots \ldots \ldots \ldots$ T8 31 


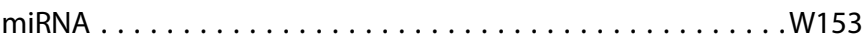
Mismatch Negativity . . . . . . . . . . M173, T39, T156, T183

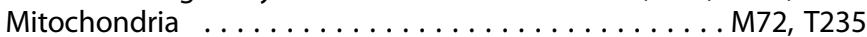
Mixed Manic Symptoms . . . . . . . . . . . . . . . .W130

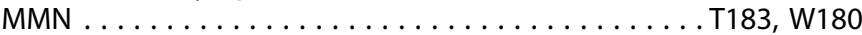

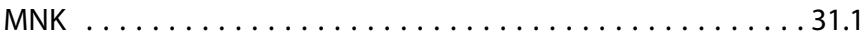

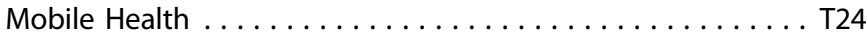

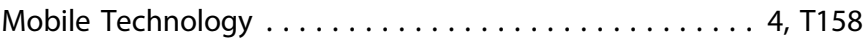
Models ....................... 30 Molecular Genetics . . . . . . . . . . . . . 7, T167, W82 Molecular Imaging . . . . . . . . . . . . . . . . . . . . . . . . T202 Molecular Mechanisms . . . . . . . . . . . 12, 19.3, T230, T233 Molecular Neuroscience . . . . . . . . . . . . . . . . . . . . 52 Monetary Reward . . . . . . . . . . . . . . . . . . T165, T248, W13 Monoamines ...................... T65, W132 Monoclonal Antibodies . . . . . . . . . . . . . . . . . . . 50.2

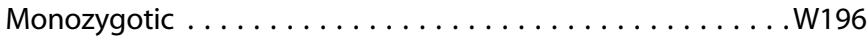
Mood . . . . . . . . . . . . . . . . . . 27, T155, W121 Mood and Anxiety Disorders ...3, 37.1, 53.1, M126, T158, W139, W217

Mood Disorder . . . . . . . . 7.1, M83, M89, M114, M224, T111, T120, T134, T152, W91, W92, W100 Mood Disorder Subtypes ...................W125 Morphine ........................W244

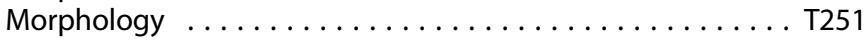
Mortality ..........................M50 Motivation ......M140, M282, T45, T168, T201, W190, W230 Mouse Behavior .....................55.1 Mouse Genetics . . . . . . . . . . . . . . . . . . . . . . . T52 Mouse Model . . ......15.2, 40.2, M13, M162, M172, M232, T70, T222, T245, W226 Movement Disorders . . ..............T170, T171

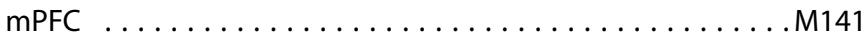

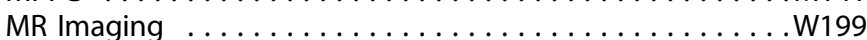
MR Spectroscopy $\ldots \ldots \ldots \ldots \ldots \ldots \ldots \ldots$ M21, M262, T205

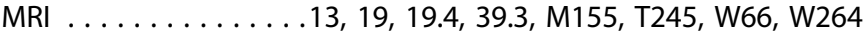

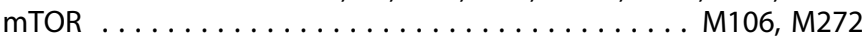

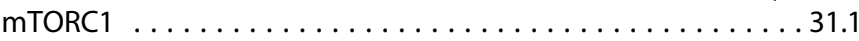

Multiplex Families . . . . . . . . . . . . . . . . . . . . . . . . . . . M201

Multi-Triggered Relapse . . . . . . . . . . . . . . . . . . . M229 Mu-Opioid Receptor . ........... T34, T125, T157, T202 Muscarinic Acetylcholine Receptor . . . . . . . . . . . . 53.4, M183

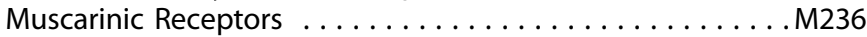

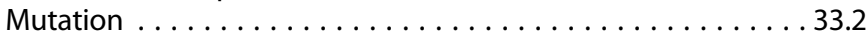

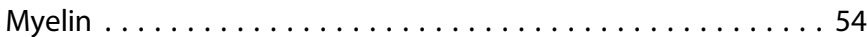

Myelin Imaging . . . . . . . . . . . . . . . . . . . M41 Myoinositol ........................ M206, W100

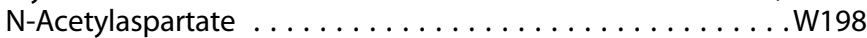
N-Acetylcysteine . . . . . . . . . . . . . . . . . . . . . . . 2.3, M180

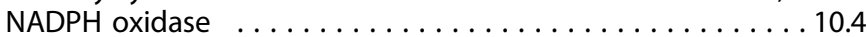

Naltrexone . . . . . . . . . . . . . . . . . . . . . T187, W253

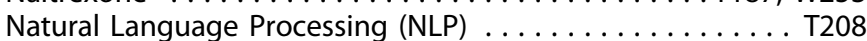

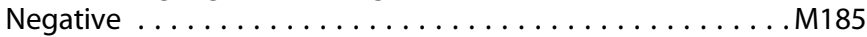

Negative Affect .................... 23.2, T79

Negative Allosteric Modulator ................ T59

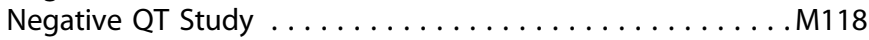

Negative Reinforcement . . . . . . . . . . . . . . 23.3

Negative Symptoms . . . . . . . . . . . . . . .M202, T210, W190

Neonatal . . . . . . . . . . . . . . . . . . . . . . . . .W58

Nest-Building ........... . . . . . . . . . . . . T147

Network Based Statistic (NBS) . . . . . . . . . . . . T77

Network-Analysis . . . . . . . . . . . . . . . . . . . T29, T235

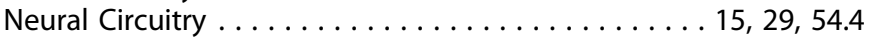

Neural Circuits ........................... . . . . .

Neural Heterogeneity . . . . . . . . . . . . . . . . 41

Neural Oscillations .....................W227

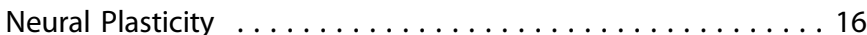

Neural Progenitor Cells . . . . . . . . . . . . . . M146, W36
Neuregulin-1 ......................W178

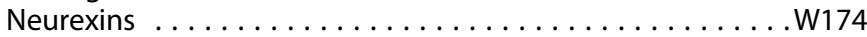

Neuroanatomy $\ldots \ldots \ldots \ldots \ldots \ldots \ldots \ldots \ldots \ldots$. . . . . . . . . .

Neurobehavioral Disorders ................. 33

Neurochemistry . . . . . . . . . . . . . . M206, T265

Neurocircuits . . . . . . . . . . . . . . . . . . . . . 41, T111

Neurocognition . . . . . . . . . . . . . . M253, W165

Neurocognitive Functioning . . . . . . . . . M155, M187

Neurodevelopment . . . . . . . . . . . . . 26.1, 26.3, 54.2, M143,

T91, T182, T245, W55, W179

Neurodevelopmental Disorders ...... 7.3, 14, M30, M37, M42, M144, M241, T97, W59, W212

Neuroeconomics . . . . . . . . . . . . . . . . . . . . . . . 18.1

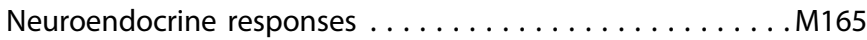

Neuroendocrinology ......................... M108

Neuroepigenetics . . . . . . . . . . . . . . . . . . . . . . . T50

Neurogenesis Enhancers .............. 58.4, W9 Neurogenetics . . . . . . . . . . . . . . .33.3, 33.4

Neuroimaging ........... 2, 33.4, 39, 56, T78, W154

Neuroimaging Biomarkers . . . . . . 2.4, 12.2, M94, W117, W207

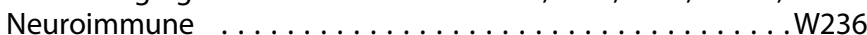

Neuroimmune Activation . . . . . . . . . . . . . . . M244

Neuroimmune Mechanisms . . . . . . . . . . . . . W179, W279

Neuroimmunology . . . . . . . . . . . M60, T130, W59, W230

Neuroinflammation .......26, 51, M51, M283, T44, W152, W267

Neurological Soft Signs . . . . . . . . . . . . . . M196

Neuromelanin . . . . . . . . . . . . . . . . . . . W264

Neuromodulation . . . . . . . . . 16.3, 17, 40.3, M47, M156

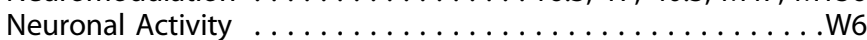

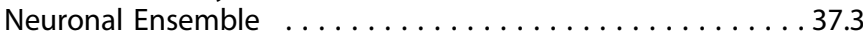

Neuronal Nitric Oxide Synthase . . . . . . . . . . . 10.2, T173, W71

Neuropathic Pain . ........... 16.4, 31, 31.1, 31.2, 31.3

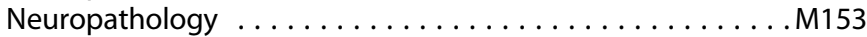

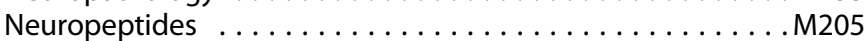

Neuropharmocology . . . . . . . . . . . . . . . . T172

Neurophysiology ......................36, W21

Neuroplasticity . . . . . . . . . . 29.4, M72, W141, W262

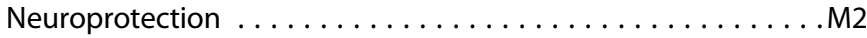

Neuropsychiatric Disorders . . . . . . . . . . . . 14, 16, 43, T162

Neuropsychiatric Symptoms (NPS) . . . . . . . . . . . T159

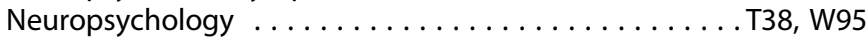

Neuropsychopharmacology ... . . . . . . . . . . . W164

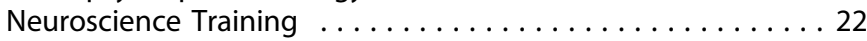

Neurosteroid . . . . . . . . . . . . . . . . . . . . . . 8, 8.2, 8.3, M242

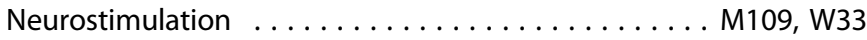

Neurosurgery ....................... T160

Neuroticism . . . . . . . . . . . . . . . . . . . . T127

Neurotransmitter Co-Release . . . . . . . . . . . . W55

Neurovascular Coupling . . . . . . . . . . . . . . . M165

New Technology ...................... 52

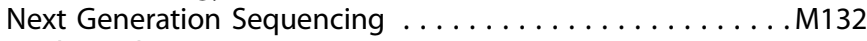

N-Glycosylation . . . . . . . . . . . . . . . . . . . . . . M179

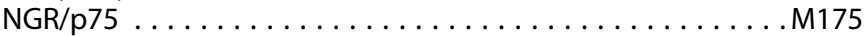

Nicotine .......... M230, M237, T58, W55, W245, W255

Nicotine Addiction ............M249, M275, T283, W163, W243

Nicotine Dependence . . . . . . . . . . . . . . . . . . . T242

Nicotinic Acetylcholine Receptors ... . . . . . . . 53.1, T87, W162

Nicotinic Receptors . . . . . . . . . . . . . . . W282

Nitrites . . . . . . . . . . . . . . . . . . . . . . . . M217

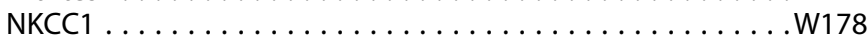

NMDA Antagonists ................. T146, W211

NMDA glutamate receptors ... . . . . . 27.1, 49.2, W243, W246

NMDA Receptor . .20.1, 34, M124, T156, T217, W84, W134, W142

NMDA-R antagonists . ................W223

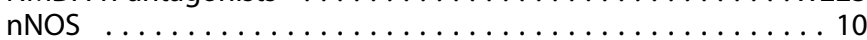

NODDI ....................... T177

Non Human Primate . . . . . . . . . . 37, M235, T37, W179, W188

Non Pharmacological Interventions . . . . . . . . . . T24 
Non-Coding DNA ..................... 11

Noncoding RNA . . . . . . . . . . . . . . . . . . 11.1

Nonhuman Primate Models . . . . . . . . . . . 37.2, 54.1, M29

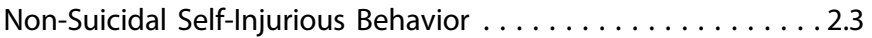

NOP Receptor Antagonist . . . . . . . . . . . . . . . . . .W25

Noradrenaline ..................... T4, T10

Norepinephrine ........................ M122

Norepinephrine Transporter Gene (SLC6A2) . . . . . . . . . . T90

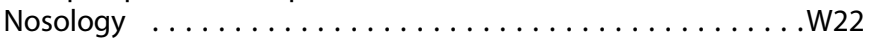

Novel Antidepressant . . . . . . . . . . . . . . . . . . T147

Novel Targets . . . . . . . . . . . . . . . . . . . . . . . . . . . 31

Novel Therapeutics . . . . . . . . . . . . . 50.3, M23, W142

Novelty Response . . . . . . . . . . . . . . . . . . . . . . . . M221

NR2B Receptor ........................... . . . . . . . . . . . . . . .

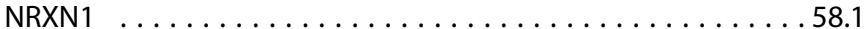

Nuclear Factor Kappa B . . . . . . . . . . . . . . . . . . . W245

Nucleus Accumbens . . . . . . . . . 1.1, 1.2, 1.3, 12.3, 35.1, 45.3,

M119, T50, T163, T219, T230, T248, T270, T282, T285, W87, W243, W273

Nucleus Accumbens Shell . . . . . . . . . . . 45.1, M264, T280

Number Needed to Harm . . . . . . . . . . . . . . . . . . . W186

Number Needed to Treat . . . . . . . . . . . . . . . W186

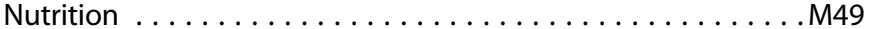

Obesity ........15, 15.4, M95, M217, T19, T100, W62, W122

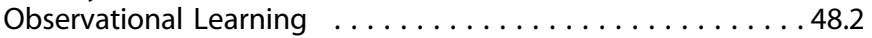

Obsession ..........................W166

Obsessive-Compulsive Disorder (OCD) . . . . . . 1.4, 2.2, 6.2, 40.2, M20, M25, M147, T65, T77, T92, T97, W10, W17,

W31, W66, W160, W165, W166

Obsessive-Compulsive Disorder (OCD) Phenotypes . . . . . . M147

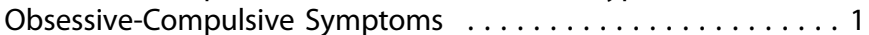

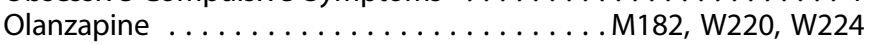

Older Adults . . . . . . . . . . . . . . . . . . . . . . T67

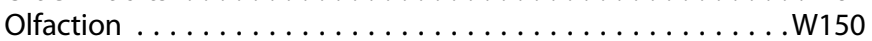

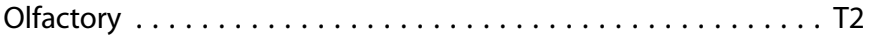

Omega-3 Fatty Acids . . . . . . . . . . . . . . M71, T107

Open Chromatin . . . . . . . . . . . . . . . . . . . . . . . . . 58.2

Open Neuroscience . . . . . . . . . . . . . . . . . . M138

Opiate Addiction . . . . . . . . . . .6.4, M268, M274, T243, W275

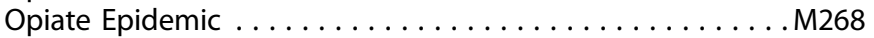

Opiates . . . . . . . . . . . . . . . . . . . . . . . . 9.2, M270

Opioid . . . . . . . . . . . . . . . . . . . . . . 18, W283

Opioid Abuse ...........M266, M271, M276, T267, W152

Opioid Addiction . . . . . . . . . . 18.1, M232, M235, M266,

T241, T248, T257, W259, W260, W266

Opioid Agonist Treatment . . . . . . . . . . . . . . . . M2.4, M266, T252

Opioid Antagonist Treatment . . . . . . . . . . . .M276, T269, W224

Opioid Dependence . 9, 23, 23.3, 23.4, M270, M271, M273, W250

Opioid Epidemic- Novel Approaches . . . . . . . . . . . . . . 9, 31

Opioid Overdose ............... T269, W253, W259

Opioid Receptors . . . . . . . . . . . . . . . . . . . . . . . . T269

Opioid Tolerance . . . . . . . . . . . . . . . . . . . M271

Opioid Treatment ...................... T259

Opioid Use Disorder . . . . . . . . . . . . . . . . . . . . T262

Opioid Withdrawal . . . . . . . . . . . . . . . . . W241

Opioids . ............ 16.4, 23.2, 50, 50.3, M265, T157

Optogenetics ........45, 45.1, M264, T22, T39, T62, T168,

W155, W239, W284

Orbitofrontal Cortex (OFC) . . . . . . . . M8, W57, W155, W165

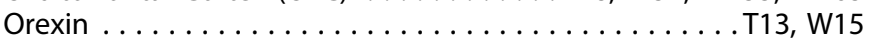

Orexin Receptor Antagonist . . . . . . . . . . . . M268, T226

Orexin System . . . . . . . . . . . . . . . . . . . . . T40, T283

Orphan Receptors . . . . . . . . . . . . . . . . . . . . . 21

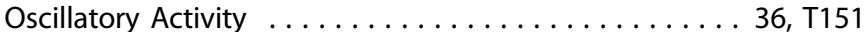

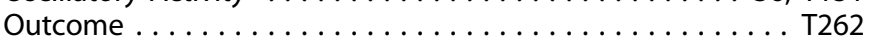

Ovarian Hormones . . . . . . . . . . . . . . . . T169

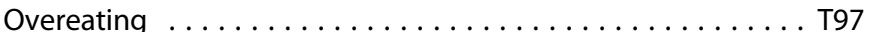

Oxidative Phosphorylation . . . . . . . . . . . . . . . . . M177
Oxidative Stress . . . . . . . . . . . . . . . . . . . W72

Oxytocin . ................ 37.2, M207, T35, W19

Oxytocin Receptor . . . . . . . . . . . . . . . . .WW233

P2X7 .................26, 26.3, W91, W92, W113

P300 ........................... T194, T205

Pain . . . . . . . . . . . . . . . . 23, W148, W168, W283

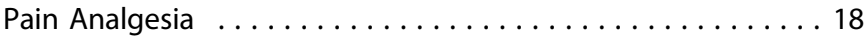

Pair Bond . . . . . . . . . . . . . . . . . . . . . . . . M163

Panic ......................... M6, W15

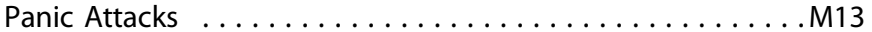

Parabrachial .......................... T62

Paranoia .............................. M194

Paraventricular Nucleus of the Thalamus . . . 45.1, 45.2, 45.3, T40

Parent - Child Dyads ... . . . . . . . . . . . 48.2, 48.4

Parvalbumin Interneurons . . . . . . . . . . 10.4, M223, T20

Parvalbumin Neurons . . . . . . . . . . . . . . . . 40.1, M177, W50

Pathophysiology ....................... . T65

Pathway Analysis ....................W75, W261

Pattern Recognition Receptors ... . . . . . . . . . . . . . M99

Pavlovian Conditioning $\ldots \ldots \ldots \ldots \ldots \ldots \ldots$. . . . . . . . T100

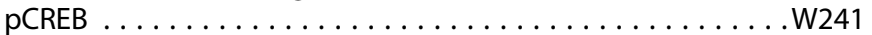

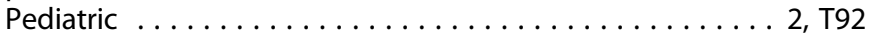

Pediatric PTSD . . . . . . . . . . . . . . . . . 48.3, W40

Peptidomics . . . . . . . . . . . . . . . . . . . . . M205

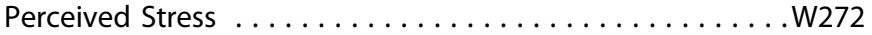

Perception .........................W63

Perceptual Learning . . . . . . . . . . . . . . . . . T193

Perfusion . . . . . . . . . . . . . . . . . . . . . M42, M45

Periaqueductal Grey (PAG) . . . . . . . . . . . . . . . . . . . W241

Perimenopausal Depression ...................108

Perinatal Stress . . . . . . . . . . . . . . . . . . W58, W59

Peripheral Biomarker . . . . . . . . . . . M89, T140, T209

Persecutory Ideation . . . . . . . . . . . . . . . . . . . . M194

Personal and Social Performance (PSP) . . . . . . . . . T114, W184

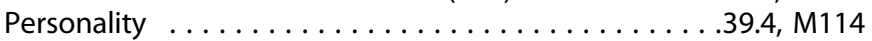

Personalized Medicine . . . . . . . . . . . . . . . M147, T124

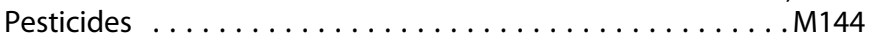

PET Imaging Study $\ldots \ldots \ldots \ldots \ldots \ldots \ldots \ldots \ldots \ldots$ T256

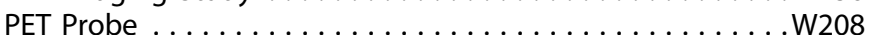

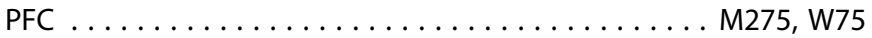

Pharmacodynamics . . . . . . . . . . . . . . . . .W219

Pharmacoegenetics ........................ . . . . . . . . .

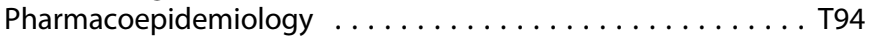

Pharmacogenetic Response . . . . . . . . . . . . . . . . . . . . . M84

Pharmacogenetics .................T123, W82

Pharmacogenomic-Guided Treatment Recommendations . . M84

Pharmacogenomics ..................... 147, W151

Pharmacokinetic and Pharmacodynamic . . . . . . . .W187

Pharmacokinetics ................... T86, W51, W221

Pharmacology .................... M85, M215

Pharmacotherapy .................. M270, M273, W23

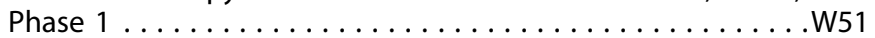

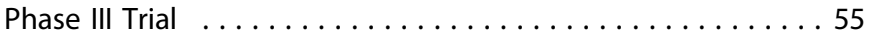

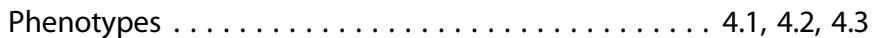

Phenotypic Screening . . . . . . . . . . . . . . . W81

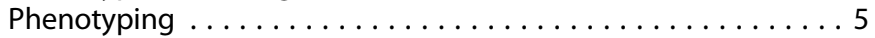

Phosphorylation $\ldots \ldots \ldots \ldots \ldots \ldots \ldots 43,43.1,43.2,43.3,43.4$

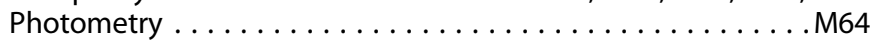

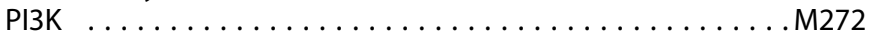

Placebo Response . . . . . . . . . . . . . . . . . . . . . . . . . . . T142

Plasma Membrane Monoamine Transporter . . . . . . .W132

Pluripotent Stem Cells . . . . . . . . . . . . . . . . .W181

Polydrug Use . . . . . . . . . . . . . . . . . . T265

Polygenic Risk Score . . . . M199, M203, T135, T196, W156, W195

Polygenic scores . . . . . . . . . . . . . . . . . . . . . . . . . . . . . . . W167, W214

Positive Allosteric Modulator . . . . . . . . . . . . M147, M148

Positive and Negative Syndrome Scale . . . . . . . . . . . . M184

Positive Symptom Factor . . . . . . . . . . . . . . M186 
Positron Emission Tomography (PET) . M14, M103, M160, M212, T203, W267, W270

Positron Emission Tomography (PET) Imaging 27, 32.1, M53, M97, M104, M134, M169, M211, M215, T37, T41, T68, T161, W113, W219 Post Traumatic Stress Disorder (PTSD) . . . . . . 3.2, 35.2, 46, 46.3, 48, 54.3, M7, M14, M16, M18, M21, M26, M27, M70, M92, M117, M134, T1, T4, T7, T9, T38, T71, T76, T79, T173, T174, T175, T262, W7, W8, W9, W12, W20, W21, W22, W23, W25, W27, W167, W170 Postmortem . . . . . . . . . . . . 43.2, T206, T271 Postmortem Brain Tissue 44.3, 52.4, M116, M177, T126, T179, W182 Postmortem Human Brain . . . . . . . . . . . . 44, 58.4, T207 Postpartum ....................... M126, W121 Postpartum Depression . . . . . . . . . . 8, 8.2, 8.3, T259 Potassium ATP channels . . . . . . . . . . . . . . . . . . .W193 Potassium Channel ........................W100

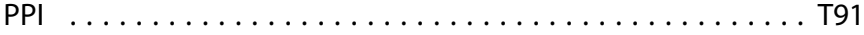
Prairie Voles ......................37, 37.3, M163

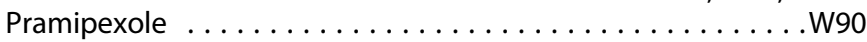

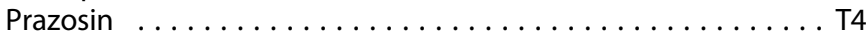
Precision Medicine . . . . . . . . . . . . . . . . . . . . . . . . . T167

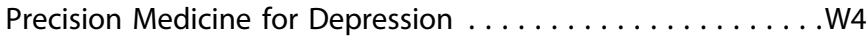
Preclinical . . . . ........................M276 Preclinical Alzheimer's Disease . . . . . . . . . . . . . M52, W2 Prediction . . . . . . . 25.1, 33.2 Prediction Error .......................... M193 Predictive Models .....................W73

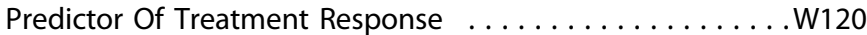
Predictors of Response . . . . . . . . . . . . . . . . M20 Prefrontal Cortex . . . . . . 40, 54, M166, M212, M234, T20, T21, T87, T279, W101, W188, W233 Pregnancy . . . . . . . . . 13.1, M85, M143, M200, T145, T227 Prelimbic . . . . . . . . . . . . . . . . . . . . . . . . . . . . . . T272 Premenstrual Dysphoric Disorder . . . . . . . . . . . . . W78

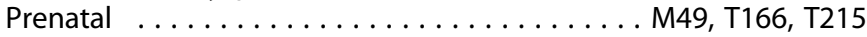
Prenatal Exposure . . . . . . . . . . 13.3, M60, T218, W49, W56

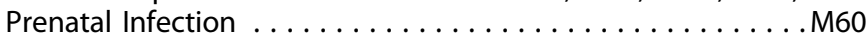
Prenatal Stress ......................... 172 Prenatal Stress-Immune Model . . . . . . . . . . . . . . . . . . . W57 Prescribing ........................... 139 Prescription Opioids . . . . . . . . . . . .23, 41.2, W229 Prevalence ........................W14 Prevention of Relapse by Voluntary Aerobic Exercise . . . . M229 Primary Auditory Cortex . . . . . . . . . . . . . . . . M173 Probabilistic Reward Learning . . . . . . . . . . . T21 Processing Speed .....................W4 Prodrug ... . . . . . . . . . . . . . . . . . . . . . . . . . W157 Proenkephalin ...................... T34 Progesterone ... . . . . . . . . . . . . . . . . . . . . . . . . M97 Pro-Inflammatory Cytokines . . . . . . . . . . . . M112 Proof of Mechanism . . . . . . . . . . . . . . . . 12.3 Protein Conformations . . . . . . . . . . . . . . . W174 Protein Dynamics . . . . . . . . . . . . . . . . . . . W174 Protein Insolubility . . . . . . . . . . . . . . . . . . . . . M176

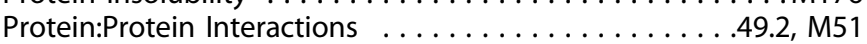
Proteomics . . . . . . . . . . . . 43.2, M13, M72, W238 Proton Magnetic Resonance Spectroscopy . . . . M90, T56, T247

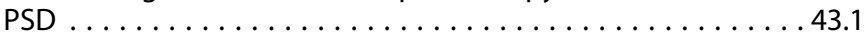

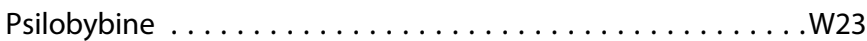
Psilocybin . . . . . . . . . . . . . . . . . . W99, W126 Psychedelic Medicine . . . . . . . . . . . .T143, T222, W99, W126

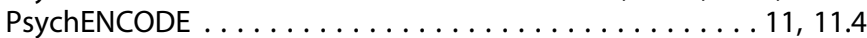

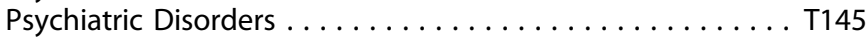

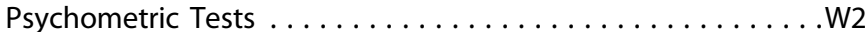
Psychomotor Speed . . . . . . . . . . . . . . . . M251, T212 Psychophysiology . . . . . . . . . . . . T241, W167, W170 Psychosis ....... 5, 24.1, 33.1, 56, M133, M182, M208, M219 Psychosis Continuum . . . . . . . . . . . . . . 56.2
Psychosis Risk . . . . . . . . . . . . . . . . . M199, M209

Psychosocial Stress . . . . . . . . . . . . . . . . T96

Psychosocial Treatment . . . . . . . . . . . . . . . . T84

Psychosomatic Medicine . . . . . . . . . . . . . . T220

Psychostimulant ..................... T266

Psychotherapy $\ldots \ldots \ldots \ldots \ldots \ldots \ldots \ldots \ldots$. . . 46.3

Psychotic Disorders ... . . . . . . M224, T41, T47, W198, W202

Psychotomimetic effects . . . . . . . . . . . . . ..W223

Psychotropic Medications . ............... M181, W66

Psyichiatric Disorders . . . . . . . . . . . . . . . W208

Puberty ... . . . . . . . . . . . . . . . . . . . . . . . M43

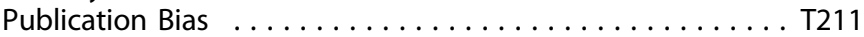

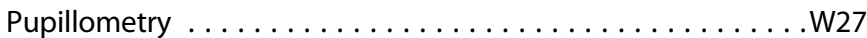

Purine .......................... T266

Pyramidal Cell . . . . . . . . . . . . . . . . . . . M177

Pyramidal Neuron . . . . . . . . . . . . . . . . . W54

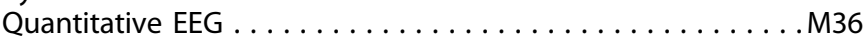

Quantitative Electroencephalography (qEEG) . . . . . M149, T136

R-(-)-Ketamine . . . . . . . . . . . . . . W131, W133

Racemic Ketamine and metabolites .......M119, M128, W133

Racial Ethnic Minority . . . . . . . . . . . . . . . . . . . . . . . . . M137

Raclopride . . . . . . . . . . . . . . . . . . . . . T243

Raman Spectroscopy ... . . . . . . . . . . . . . . T209

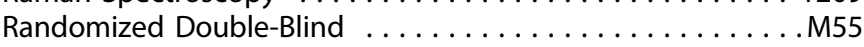

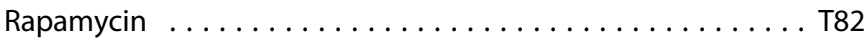
Rapastinel ....................... M124, M269, W84

Rapid Antidepressant . . . . . . . . . . . . 20.1, W84

Rapid Antidepressant Effects . . . . . . . . . . . . . 34.4

Rapid Depression Treatment . . . . . . . . . . . . . . . M123

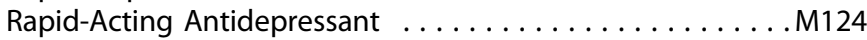

Rare Disorders ...................... 33

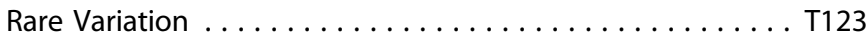

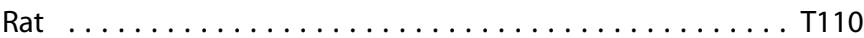

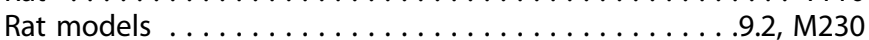

Rater Reliability . . . . . . . . . . . . . . . . W88

Rating Scales .......................W46, W143

Readmission risk ..................... T208

Real-Time fMRI Neurofeedback . . . . . . . . 17, 17.3, M87

Reappraisal ........................ T73

Recent Onset Psychosis . . . . . . . . . . . . . . . T203

Receptor Heteromerization . . . . . . . . . . . . . . . T231

Receptor Internalization Trafficking, ... . . . . . . . . . . M239

Receptor Occupancy ....................W113

Reciprocity ...................... T93

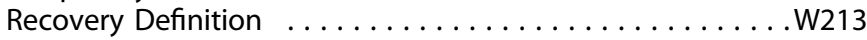

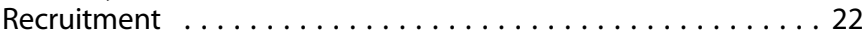

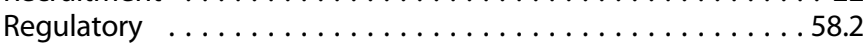

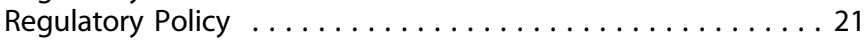

Reinforcement . . . . . . . . . . . . . . . . . . . M282, T264

Reinforcement learning . . . . . . . . T21, T89, T142, W190

Reinforcement-based decision-making . . . . . . . . .W238

Reinstatement . . . . . . . . . . . . . . . . . . . . 50.4, T223, T224

Relapse ..................... T113, W232

Relapse and Treatment Outcome . . . . . . . . . . 18.1

Relapse Biomarkers . . . . . . . . . . . . . . . . . M208, M252, T55

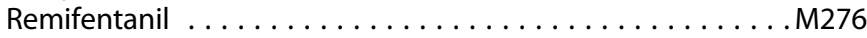

Remote Memory ..................... T3

Repastinel .......................... 34.2

Repetitive Transcranial Magnetic Stimulation (rTMS) . . M47, T273

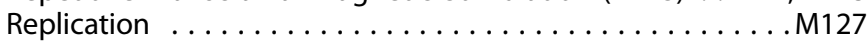

Reproducibility ........................ M127

Research Domain Criteria (RDoC) . . . . . . . . M155, T23, W94

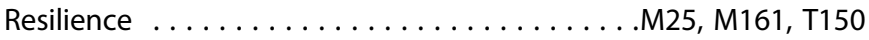

Respiration ..................... T10

Response Inhibition ............... T16, T261, W34

Resting State .................... T186

Resting State fMRI . . . . . . . 2.3, 39.1, M79, M80, M97, M100,

M136, M230, M248, M257, T25, T77, T90 
Resting State Functional Connectivity ... M27, M36, M43, M101, M102, M136, M158, M164, M208, M231, M255, T35, T54, T56, T67, T74, T76, T92, T98, T152, T186, T197, T204, W12, W58, W109

Resting State Networks .M96

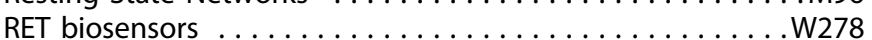

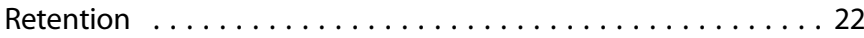
Retina .......................W86

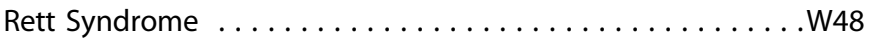
Reversal Learning . . . . . . . . . . . . . . . . M30, W70 Reward ......19.4, 41, M66, T54, T62, T265, W61, W155, W190 Reward and Aversion . . . . . . . . . . 16.4, 41.3, W168 Reward Circuitry . . . . . . . . . . . . 15.3, 45.2, M88, T176 Reward Functioning ................... T152 Reward Learning . . . . . . . . . . . . . . . .T22, T64 Reward Neural Circuitry . . . . . . . . . . . . . . . . . 35 Reward Processing ................... M31, T190

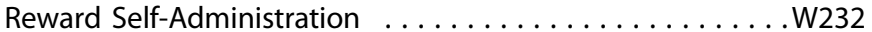
Reward Sensitivity ......................65 Reward-Based Decision-Making . . . . . . . . . . M64, M194

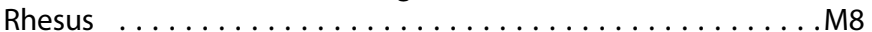

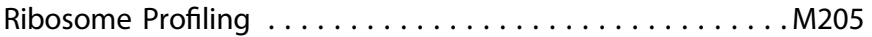
Right Temporo-Parietal Junction . . . . . . . . . . . . . . . M207 Risk . . . . . . . . . . . . . . . . . . . . . . . . . . . . 24.4, T94

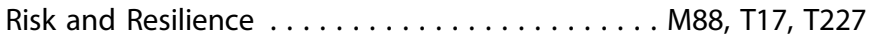
Risk Factors . . . . . . . . . . . . . . . . . . . . . . . . W214 Risk for Social Anxiety Disorder . . . . . . . . . . . M17 Risk-Taking . . . . . . . . . . . . . . . . . . . . M81 Risky Decision-Making . . . . 41.3, M33, M245, M254, T239, T276 RMTg ......................... M141, W237 RNA Sequencing . . . . . . . . . . . . . .44, 44.4, M143, T33, T206, T235, W78, W244

RNA Splicing ...........................44.3 RNAscope Fluorescence in Situ Hybridization . . . . . . T182, T207

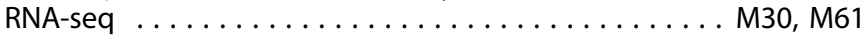

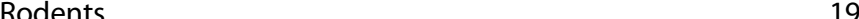
Safety . . . . . . . . . . . . . . . . . M55, M118, W51, W221

Salience Network . . . . . . . . . . . . . . . . . . M259, T89 Samidorphan .......................W85, W136 Satiation . . . ....................W61

Schizophrenia . . . .11.3, 12.2, 12.4, 25, 25.3, 25.4, 26.1, 29.1, 29.4, $32,38,43.2,43.4,49,49.4,58.1,58.3, \mathrm{M} 172$, M176, M177, M178, M180, M184, M185, M188, M196, M197, M201, M202, M206, M207, M210, M215, M216, M217, T40, T42, T43, T44, T46, T179, T183, T188, T191, T193, T196, T201, T202, T210, T212, T213, W36, W182, W184, W186, W193, W195, W197, W200, W214, W221, W222, W224

Schizophrenia Genetics . . . . . . . . . . . . . M179, W194 Schizophrenia Negative Symptoms . . . . . . . . . . . . . . . . . T45 Schizophrenia Novel Treatment . . . . . M183, M186, M190, T184, T185, T187, T189, T213, W183, W187, W219 Schizophrenia Spectrum Disorders . . . . . . . . . . . . . 56.3 Schizophrenia Subtypes ................25, W217 Schizophrenia-like Behavior . . . . . . . . . . . 49.3, M222, T218 Schizotypal Personality Disorder ..............W195

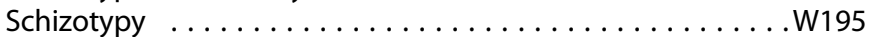
Schnurri-2 Knockout Mice . . . . . . . . . . . . . . . . . . W180

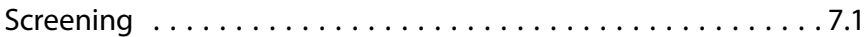
Seasonal Affective Disorder . . . . . . . . . . . . . . . . M68 Seizures ..................... 21, T160 Self-Administration . . . . . . . M225, M269, T278, T267, W229, W234, W237, W274

Self-Efficacy . . . . . . . . . . . . . . . . . . . . . . . . M81

Self-Injurious Behavior ..................M162

Self-Referential . . . . . . . . . . . . . . . . . . . . . . W269

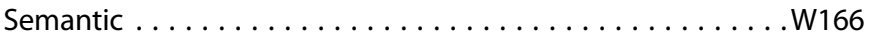

Senescence Associated Secretory Phenotype . . . . . . . . .W1

Sensitive Period ........................W149
Sensitization . . . . . . . . . . . . . . . . . . . . . . M240

Sequencing . . ....................11.3, M202

Sequential Parallel Clinical Design . . . . . . . . . . . . . . T138

Serious Mental Illness .................... T196

Serotonergic System . . . . . . . . . . . . . . . . . . . . . . . T28

Serotonin .......M6, M111, M157, M284, T106, W139, W149

Serotonin 1A Receptor .............M284, T155, W28

Serotonin 5-HT2A Receptor . . . . . . . . . . . . . M226

Serotonin 5-HT2C Receptor . . . . . . . . . . 50, M239, W275

Serotonin And Norepinephrine Reuptake Inhibitor . . M103, W69

Serve And Return . . . . . . . . . . . . . . . . . . . . . T93

Sex .............................. 89

Sex Differences . . . . . . . . 53.3, M9, M43, M110, M127, M128, M134, W138, W258, M281, T33, T60, T83, T111, T147, T151, T165, T218, T267, T274, W5, W30, W57, W59, W76, W132, W164, W229, W234, W272, W274,

W280, W281

Sex Hormones . . . . . . . . . . . . . . . M128, T23, W122, W280

Sex Steroids . . . . . . . . . . . . . . . . . . . T112

Shared Emotional Affect ...................99

Short-Term Clinical Studies . . . . . . . . . . . . . . . . . . . . M183

Signal Transduction ...................W181

Signaling Networks . . . . . . . . . . . . . . . .43.1, M51

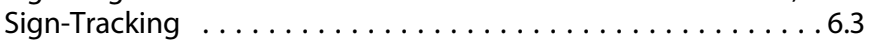

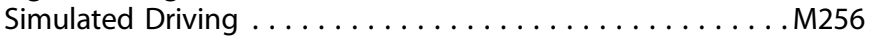

Simultaneous PET-MR . . . . . . . . . . . . . . . . . . W145

Single-cell RNA sequencing . . . . . . . 5 52.3, W8, W182, W261

Single-Cell Sequencing . . . . . . . . . . . . . . . . 52, 52.4

Single-Nucleus . . . . . . . . . . . . . . . . . . . . . . . T206

Skin Conductance Responses . . . . . . . . . . . .48.2, T5, W21

SLC39A8 ... . . . . . . . . . . . . . . . . . . . ... M179

Sleep . . . . . . . . . . . . . 1, 10.2, M36, M78, T219, W86, W142

Sleep Deprivation .....................M36

Sleep Disturbance . . . . . . . . . . . . . . . . . . . . . . . . 1.3, M31, T158

Sleep-Dependent Learning . . . . . . . . . . . . . . . T193

Slow Wave Activity . . . . . . . . . . . . . . . . . . . . . 10.2

Slow Wave Sleep . . . . . . . . . . . . . . . . . . 10.2, 10.4

Slow-Wave Sleep ....................... 10

Smartphone-Based App ...................... . . . . . . .

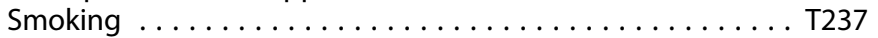

Smoking Cessation . . . . . . . . . . . . . . . . . . . W251

SNP . . . . . . . . . . . . . . . . . . . . . . . . . . M239, T197

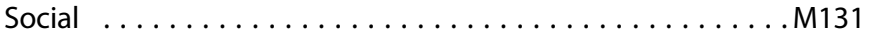

Social Anxiety . . . . . . . . . . . . . . T88, W13, W32

Social Anxiety Disorder . . . . . . . . . . . . . . . . M254

Social Behavior . ........37, 37.2, 48.4, M30, M163, T57, T83, W24, W50, W228

Social Cognition ..............56.2, 56.3, M188, M191, T24, W121, W256, W257

Social Defeat Stress . . . . . . . . 1.1, M28, M66, M73, T102, T225

Social Function ......................... 56

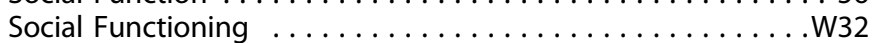

Social Processing ......................... 33.1

Social Reward ......................17, M254, W13

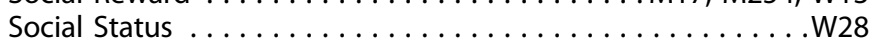

Social Stimuli . . . . . . . . . . . . . . . . . . . . M254

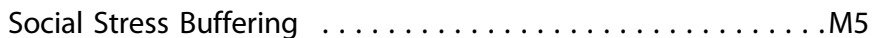

Social Withdrawal . . . . . . . . . . . . . . . . . . M220

Soluble Epoxide Hydrolase . . . . . . . . . . . . . . . . . . . . . T99

Somatomotor .......................W158

Somatosensory Network . . . . . . . . . . . . . . . . T101

Somatosensory Processing . . . . . . . . . . . . . . . . W24

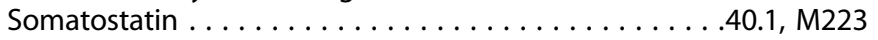

Spatial Memory .........................455

Spinogenesis . . . . . . . . . . . . . . . . . . . . . . . . . . . 16.3

State Dependence ...................W158

Stem Cells . . . . . . . . . . . . . . . . . . . . . . . . . . . . .W36

STG . . . . . . . . . . . . . . . . . . . . . . . . . M206 
Stimulant Dependence

M231

Stopping

. T16

Stress . . . . . . . . . . . . . 34.2, 52.3, M69, M129, T150, W76

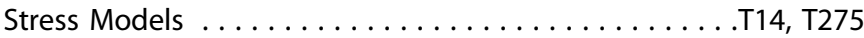

Stress Reactivity . . . . . . . . . . . . . . 15.3, M39, M244

Stress Resilience $\ldots \ldots \ldots \ldots \ldots \ldots \ldots \ldots \ldots \ldots \ldots \ldots$. . . . . . . . . . . . . . . . . . .

Stress Response Circuitry . . . . . . . . . . . . . M110

Stress-Immune Dysregulation . . . . . . . . . . . . . . W57

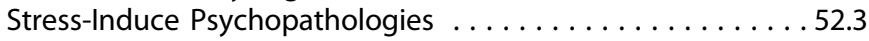

Striatal Pathways . . . . . . . . . . . . . . . . . M208

Striatum . . . . . . . . . . . . . . . . . . . . 7.2, T64, T280

Structural MRI . . . . . . . . 19.2, 25.2, 25.3, M19, T38, T242, W107, W114, W203

Structural Neuroimaging . . . . . . . . . . . 24.4, 39.2, W111

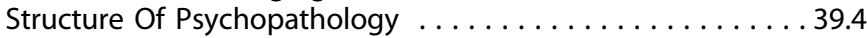

Subcallosal Cingulate .................... M80

Subcortical Shape Analysis . . . . . . . . . . . . M58, T104

Subgenual Cingulate Cortex . . . . . . . . . . . . T133, T206

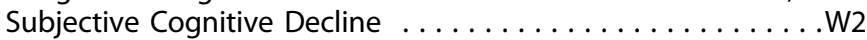

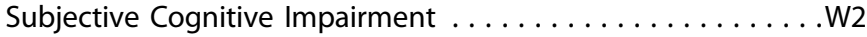

Subjective Response . . . . . . . . . . . . . . . . . . . . . . . . W276

Substance Abuse .................. M241, M283

Substance Abuse Disorders . . . . . . . . . . . 53, T249, W139

Substance Use Disorder . . . . . . . . . . . . . . . . . M266

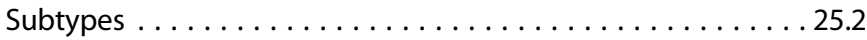

Suicidal Behavior ................W173, W214

Suicidal Ideation ...................M14, T118

Suicidal Ideation And Behavior Assessment Tool . . . . . . . W88

Suicidality .................. M48, T140, W103, W134

Suicide ..... 4 4.1, 4.2, M61, M70, M86, M89, M113, T23,

T25, T122, W93

Suicide Assessment $\ldots \ldots \ldots \ldots \ldots \ldots \ldots \ldots \ldots$. . . . . W . W93

Suicide Prediction . . . . . . . . . . . . . . . . . . M101, W73

Support Vector Machine (SVM) . . . . . . . . . . . T57, W107

susceptibility . ...................... T150

Sustained Antidepressant . . . . . . . . . . . . . . . . . . T148

Sustained Remission . . . . . . . . . . . . . . . . . . . . . . T141

Sympathetic Nervous System . . . . . . . . . . . . . . W124

Symptomatology . . . . . . . . . . . . . . . . . . . . . M100

Symptoms . . . . . . . . . . . . . . . . . . . . . . M185, W215

Synapse . ...............26.1, 34.2, 43, 49, 49.3

Synaptic Aberrations . . . . . . . . . . . . . . . 12.4, 43.4, 49.4

Synaptic Density . . . . . . . . . . . . . . . . . M75, T256

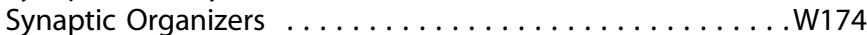

Synaptic Plasicity . . . . . . . . . . . . . . . . . W147

Synaptic Plasticity . . . . . . . . 19.3, 27, 27.2, 27.3, 31.3,

$41.2,43.4, \mathrm{~T} 49, \mathrm{~T} 270, \mathrm{~W} 243$

Synaptic Protein Interaction Networks . . . . . . . . . . W174 Synchrony . . . . . . . . . . . . . . . . . . . . T150

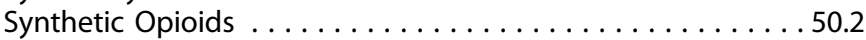

Synthetic Psychoactive Cathinones . . . . . . . . . M233

TAK-653 . . . . . . . . . . . . . . . . . . . . . . . . . . . . M148

Tardive Dyskinesia . . . . . . . . . . . . . . . . . . T170, T171

Target Engagement . . . . . . . . . . . . 12.2, W185, W189

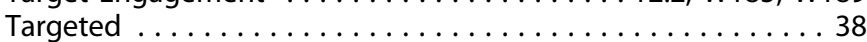

Targeted Cognitive Training . . . . . . . . . . . . . . T183

Task-Based Functional Connectivity . . . . . 54.3, M259, T26, T195,

W275

Taste . . . . . . . . . . . . . . . . . . . . M57, W63

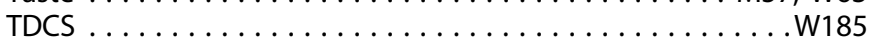

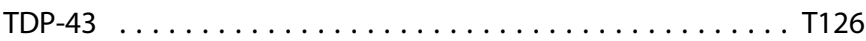

Technology . . . . . . . . . . . . . . . . . . . . 58.3

Telemedicine ....................... T164

Teletherapy . . . . . . . . . . . . . . . . . . . . . . . T164

Telomere . . . . . . . . . . . . . . . . . . . . . . . . . M86

Temperament .................... M114, M227, W217

Temperature Regulation . . . . . . . . . . . . . . . . . . . . M140

Testosterone ..........................W67

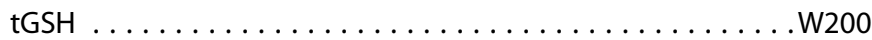

Thalamus $\ldots \ldots \ldots \ldots \ldots \ldots \ldots \ldots \ldots \ldots \ldots \ldots$ T260, T283

THC . . . . . . . . . M267, T54, T215, T263, W237, W261

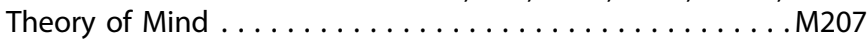

Theta .................... 36, M193, W191

Theta Burst Transcranial Magnetic Stimulation . . . . . . . . W12

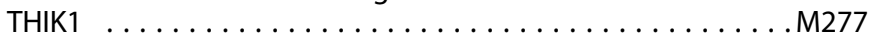

Thorough QT study $\ldots \ldots \ldots \ldots \ldots \ldots \ldots \ldots \ldots \ldots \ldots \ldots$

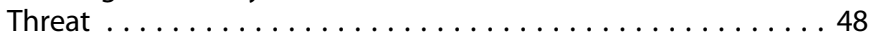

Threat Conditioning $\ldots \ldots \ldots \ldots \ldots \ldots \ldots .3,48.3$

Threat Faces ... . . . . . . . . . . . . . . . . . . . . M24, M191

Threat of Shock . . . . . . . . . . . . . . . . T240

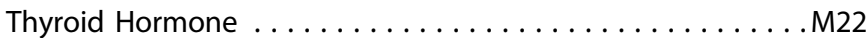

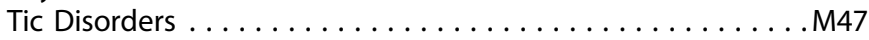

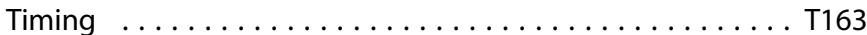

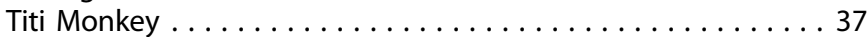

TMS ................ M3, M149, T55, T261, W141

TMS EEG . . . . . . . . . . . . . . . . . . . . . T153

TMS Targeting $\ldots \ldots \ldots \ldots \ldots \ldots \ldots \ldots \ldots \ldots \ldots \ldots \ldots \ldots$

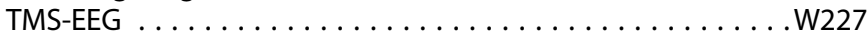

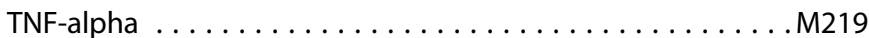

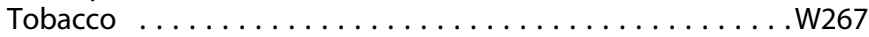

Tobacco Smoking . . . . . . . . . . . . . . . M248, W251

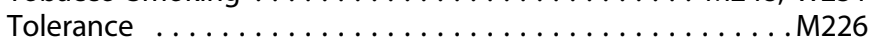

Toll-Like receptors (TLRs) $\ldots \ldots \ldots \ldots \ldots \ldots \ldots$. . . . . . . . . . . . . . . . 242

Top-Down Control . . . . . . . . . . . . . . . . . . . . M24

Touchscreen . ...................... T121

Tourette syndrome $\ldots \ldots \ldots \ldots \ldots \ldots \ldots \ldots$. . . . . . . . . .

Toxins . . . . . . . . . . . . . . . . . . . . . . . . . . . . . . M144

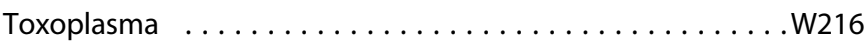

Tract Tracing . . . . . . . . . . . . . . . . . . . . . . . . W75

Transcranial Direct Current Stimulation . . . . . . . . . M213

Transcranial Magnetic Stimulation . . . . . . . . . . T137, T172

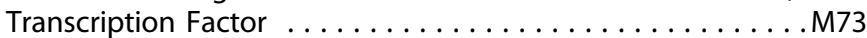

Transcription Imputation $\ldots \ldots \ldots \ldots \ldots \ldots \ldots \ldots \ldots \ldots$ 18

Transcriptional Profiling ..................W244

Transcriptome ................. 11.1, T135, T162

Transcriptomics . . . . . . . . . 7.2, 33.4, 52, 52.4, M238

transcutaneous auricular vagus nerve stimulation (taVNS) . M110

Transdiagnostic ...................... T137

Transdifferentiation ...................W212

Transgenic Mice ......................... 1.3

Transient receptor potential (TRP) A1 . . . . . . . . M140

Translational Approaches To Drug Development . . . . . . . . 12

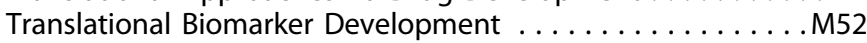

Translational Neuroscience . . . . . . . . . . . . . 6.3, 40, T68

Translational Research ..............6, 14, 27, W7

Translocator Protein .................. T44

Trauma ............................W16

Trauma Exposure .................. T196, W19

Traumatic Brain Injury (TBI) . . . . . . M92, M113, M153, M283,

T254, W176

Traumatic Memories . ...................M22

Treatment . . . . . . . . . . . . . . . . 29, M78, W33

Treatment Access . . . . . . . . . . . . . . . . . . . . . . T134

Treatment Markers ....................... 2.4

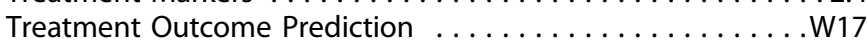

treatment resistant . . . . . . . . . . . . . . . . T144

treatment resistant cohort . . . . . . . . . . . . . . . . M115

Treatment Resistant Depression . . M84, M106, M148, T115, T116,

T128, W89, W98

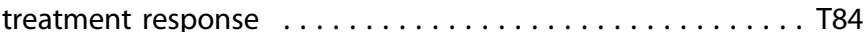

Treatment-Naive ... . . . . . . . . . . . . . . . . . T247

Treatment-Response . . . . . . . . . . . . . 2, M203, W205

Tretment Response . . . . . . . . . . . . . . . . . . . . . . M182

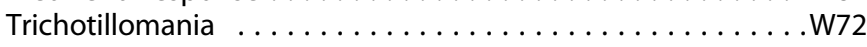

TSPO . . . . . . . . . . . . . . . . . . . . . . . . . . . . . T246

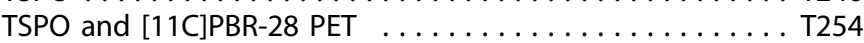


Twins

.W196

Two Photon Microscopy .

$40.4,45.2$

Two-Photon

16.1, T39

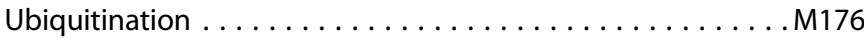

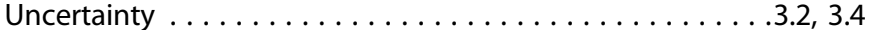

Unconditioned Responses ................. T5

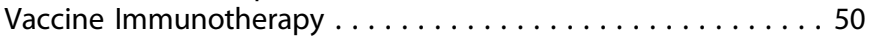

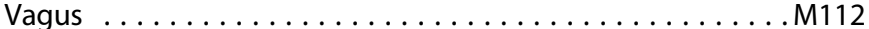

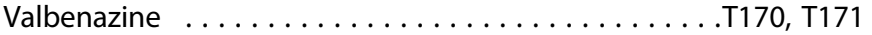

Validation .......................W88

Valproic Acid ....................... T82

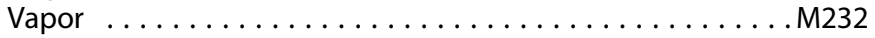

Varenicline ..........................246

Vascular ........................W114

Vasopressin 1A Receptor Antagonist .............W74

Ventral Hippocampus . . . . . . . . . . . . . . . . . . . . . T155, W70

Ventral Pallidum ...................... 41.1

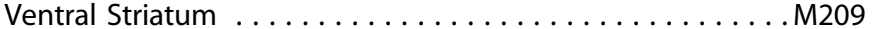

Ventral Tegmental Area (VTA) . . M62, M64, M105, M123, M126,

M277, T277, W35, W244

Ventromedial Prefrontal Cortex . . . . . . . . . . . . . . . . T272

Verbal Episodic Memory . . . . . . . . . . . . . . . . . M192

Veterans . . . . . . . . . . . . . . . . . . . . M92, W172

Vicarious Learning . . . . . . . . . . . . . . . . . 48.3

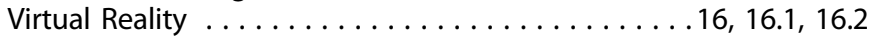

Visceral Obesity .......................W199

Visual .......................W176

Visual Attention ...................... T90

Visual Cortex . . . . . . . . . . . . . . . . . . . . . . . . . . . . . . . T87

Visual Information Processing $\ldots \ldots \ldots \ldots \ldots \ldots \ldots \ldots$ W11

Visual Memory ............................. T90

Visual Perception ......................24
Visuospatial Ability . . . . . . . . . . . . . . . . . . . . . . . . M164

Visuospatial Working Memory .............T182, T190

Voltage-Gated Calcium Channel . . . . . .....M146, T217, W218

Voltammetry ........................W284

Volume ................................. T199

Voxel-Based Morphometry (VBM) . . . . . . W40, W235, W257

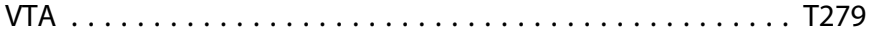

Vulnerability ................. T110, W234, W238

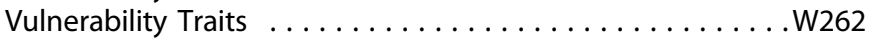

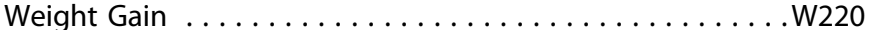

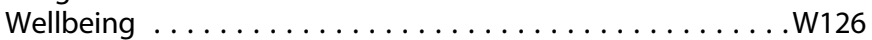

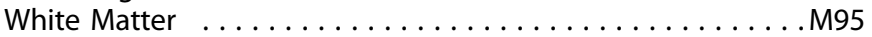

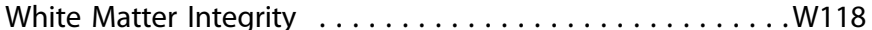

White Matter Neurons . . . . . . . . . . . . . . . . . 10.4

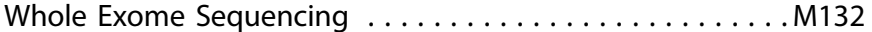

Whole Genome Sequencing . . . . . . . . . . . . . . W192

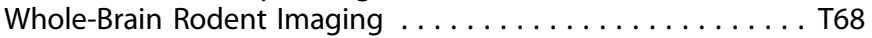

Whole-Genome .........................11.3, M202

Williams Syndrome . . . . . . . . . . . . . . . . 33.3, M41

Withdrawal . ................M270, M273, T43, T226

Within Person Variance .................. 32

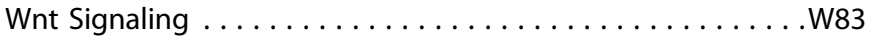

Women's Mental Health ..................T23, T74, T145

Word Embedding Clustering . . . . . . . . . . . . . W166

Workforce ...................... 22

Working Memory . . . . . . . 40.1, M3, M171, M192, M223, T26,

T195, T261, W5, W159, W191, W218, W277

World Trade Center Responders . . . . . . . . . . . W20

Worry ........................... T73

Young Adults .....................W34

Youth .....................24.4, 48.3, 54.4, W106

$\beta$-arrestin . . . . . . . . . . . . . . . . 43.3 